\title{
The OmniTread OT-4 Serpentine Robot-Design and Performance
}

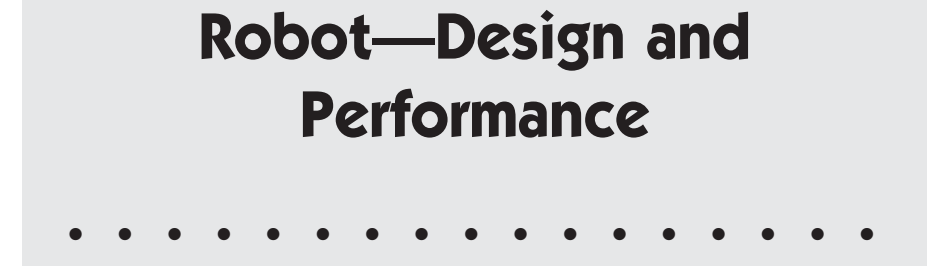

\author{
Johann Borenstein, \\ Malik Hansen, and Adam Borrell \\ The University of Michigan \\ 2260 Hayward Street \\ Ann Arbor, MI 48109 \\ e-mail: johannb@umich.edu \\ e-mail: hansenm@umich.edu \\ e-mail: aborrell@umich.edu \\ Received 25 September 2006; accepted 21 February 2007
}

\begin{abstract}
Serpentine robots are slender, multi-segmented vehicles designed to provide greater mobility than conventional mobile robots. Serpentine robots are ideally suited for urban search and rescue, military intelligence gathering, and inspection tasks in hazardous or inaccessible environments. One such serpentine robot, developed at the University of Michigan, is the "OmniTread OT-4." The OT-4 comprises seven segments, which are linked to each other by six joints. The OT-4 can climb over obstacles that are much higher than the robot itself, propel itself inside pipes of different diameters, and traverse difficult terrain, such as rocks or the rubble of a collapsed structure. The foremost and unique design characteristic of the OT- 4 is the use of pneumatic bellows to actuate the joints. The pneumatic bellows allow the simultaneous control of position and stiffness for each joint. Controllable stiffness is important in serpentine robots, which require stiff joints to cross gaps and compliant joints to conform to rough terrain for effective propulsion. Another unique feature of the OmniTread design is the coverage of all four sides of each segment with drive tracks. This design makes the robot indifferent to rollovers, which are bound to happen when the slender bodies of serpentine robots travel over rugged terrain. This paper describes the OmniTread concept and some of its technical features in some detail. In the Experiment Results Section, photographs of successful obstacle traverses illustrate the abilities of the OT-4. (c) 2007 Wiley Periodicals, Inc.
\end{abstract}

Parts of this paper were presented at the 2006 International Joint Topical Meeting: "Sharing Solutions for Emergencies and Hazardous Environments," Feb. 12-15, 2006, Salt Lake City, UT. Other parts of this paper were presented at the SPIE Defense and Security Conference, Unmanned Systems Technology IX, Orlando, FL, April 9-13, 2007

Journal of Field Robotics 24(7), 601-621 (2007) C 2007 Wiley Periodicals, Inc.

Published online in Wiley InterScience (muw.interscience.wiley.com). • DOI: 10.1002/rob.20196 


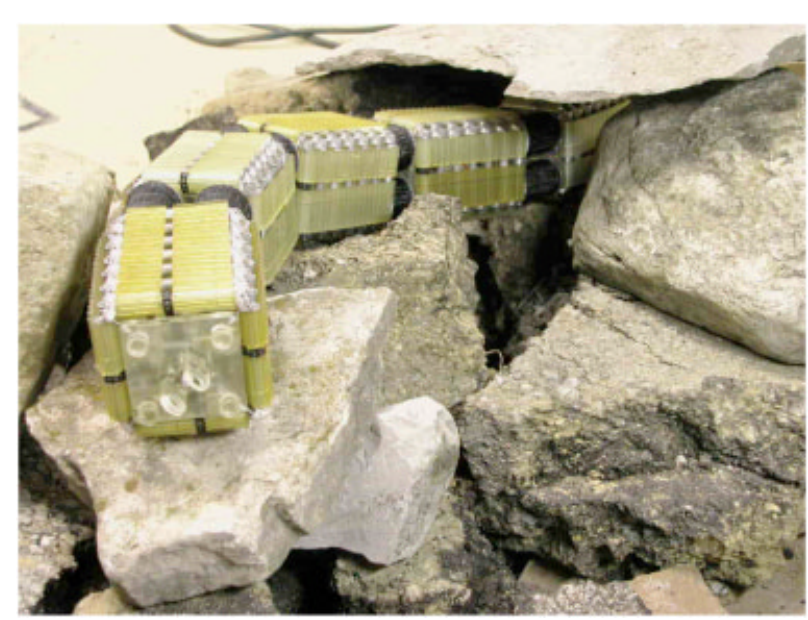

Figure 1. The OmniTread OT-4 serpentine robot slithering out of a crevice under a pile of rocks.

\section{INTRODUCTION}

Urban search and rescue, military intelligence gathering, and certain industrial inspections tasks in hazardous environments have one need in common: small-sized mobile robots that can travel across the rubble of a collapsed building, squeeze through small crawl-spaces, and slither into small openings. One species of mobile robots that promises to deliver such hypermobility is the so-called serpentine or snake robot (see Figure 1).

Serpentine robots typically comprise four or more rigid segments that are connected by 2- or 3-degree-of-freedom (DOF) joints. The terms "serpentine robot," "snake robot," and "snakebot" are used in the scientific literature interchangeably, they all mean the same thing.

One major difference within the family of serpentine robots is the type of propulsion used.

Undulating-This type of robot derives propulsion from undulations (a wavelike motion of the joints only), that is, it uses no driven wheels, legs, or tracks for propulsion. Typically, undulating robots are comprised of many segments.

Active Skin-This type of robot derives propulsion from wheels, legs, or tracks. Joints connecting the segments may be either powered or unpowered. Some researchers use the term "active skin" to describe this type of serpentine robot. These robots typically comprise fewer segments than undulating ro- bots. The OmniTread robot that is the subject of this paper has tracks and it is thus a member of the "active skin" type of serpentine robots.

Undulating robots have been attracting the attention of researchers since the 1970s. Around that time Shigeo Hirose from the Tokyo Institute of Technology developed his Active Cord Mechanism, which mimicked snake movements. The latest incarnation of this idea is the ACM-R3 robot, which is capable of performing new types of 3-D undulations [Mori \& Hirose, 2002].

In the 1990s, research on undulating robots increased dramatically as documented by Dowling (1997). Muth and Grant (2000) developed the MOCASIN II pipe crawler. This snakelike robot uses joint actuators for active propulsion while actuators embedded in the segments are used for holding consecutive links in place. Another undulating robot that uses pneumatic power for actuating its joints is the slime robot (SR) developed by Ohno and Hirose (2000). Metal bellows used in the initial prototype were exchanged for bridle bellows in the latest version, called SSR-II [Aoki, Ohno \& Hirose, 2002]. Choset and his group at Carnegie Mellon University (CMU) produced a promising undulating robot [Choset, 2005], although formal papers have not yet been published.

The focus of this paper, however, is on active skin robots. The first active skin serpentine robot, called KR-I, was introduced by Hirose and Morishima (1990) and the improved version KR-II was presented by Hirose et al. (1991). The KR-I was large and heavy, weighing in at $350 \mathrm{~kg}$.

More recently, Klaassen and Paap (1999) and Paap et al. (2000) at the German Institute for System Design Technology (GMD) developed the Snake2 vehicle, which contains six active segments and a head. Each round segment has an array of 12 electrically driven wheels evenly spaced around its periphery. These wheels provide propulsion regardless of the vehicles orientation (i.e., its roll angle). Segments are interconnected by universal joints that are actuated by three additional electric motors through strings.

While wheeled serpentine robots can work well inside of smooth-walled pipes, more rugged terrain requires tracked propulsion. To this effect Takayama and Hirose (2000) developed the Souryu I crawler, which consists of three segments. Each segment is driven by a pair of tracks, which are all powered simultaneously by a single motor, located in the center segment. Torque is provided to the two distal seg- 


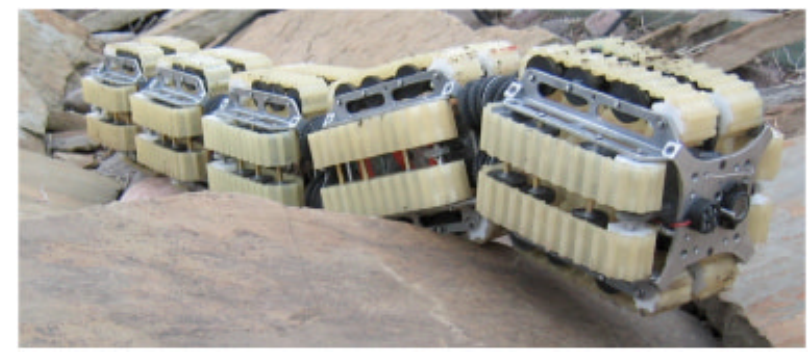

Figure 2. Our earlier-developed and larger OmniTread OT-8 can fit through an 8 -inch diameter hole.

ments through a rotary shaft and universal joints. Each distal segment is connected to the center segment by a special 2-DOF joint mechanism, which is actuated by two lead screws driven by two electric motors.

Osuka and Kitajima (2003) developed MOIRA, a serpentine robot that is strikingly similar to our OmniTread (although our efforts are independent). MOIRA comprises four segments, and each segment has two longitudinal tracks on each of its four sides, for a total of eight tracks per segment. The 2-DOF joints between segments are actuated by pneumatic cylinders. We believe that the bellows-based joint actuators used in our OmniTread have a substantial advantage over a cylinder-based design, because the bellows are more compact and don't require any space in the segments.

A different concept, using unpowered joints, was introduced by Kimura and Hirose (2000) at the Tokyo Institute of Technology. That robot, called Genbu, is probably the only serpentine robot with unpowered joints. Another robot incorporating a combination of passive and active joints as well as independently driven and coupled segments is KOHGA, developed by Kamegawa et al. (2004). This robot implements a smart design feature: Besides a camera in the front segment there is a second camera in the tail section that can be pointed forward, in the way a scorpion points its tail forward and over-head. This "tailview" greatly helps with teleoperation of the robot.

The subject of this paper is the OmniTread Model OT-4. The OT-4 is the successor to our earlierdeveloped OmniTread OT-8, shown in Figure 2. The OT-8 is so designated because it can fit through a hole 8 in. in diameter, while the OT-4 can fit through a hole

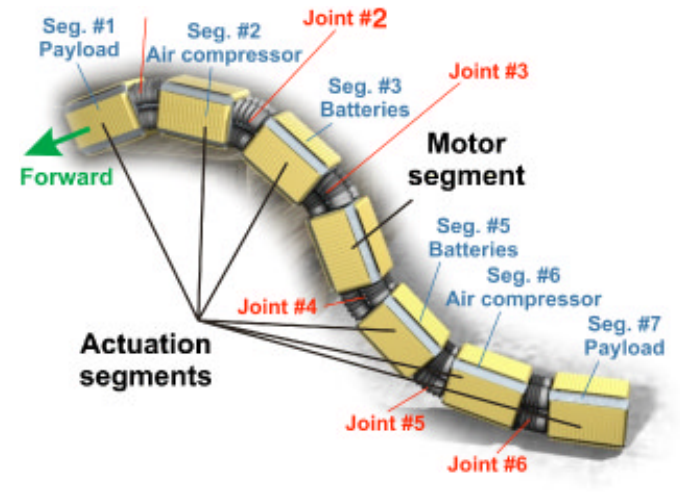

Figure 3. Nomenclature and functions for segments and joints in the OT-4.

4 in. in diameter. Our earlier paper [Granosik, Hansen \& Borenstein, 2005] describes the OT-8 in detail.

One problem with most serpentine robots is that they require many human operators for their many degrees of freedom. In the case of the OT-4, three human operators are needed. Ongoing work in artificial intelligence, notably the so-called "7G" system that is being developed by William Hutchison and Betsy Constantine, aims at reducing the number of human operators to just one. The 7G work, as it pertains to serpentine robots, has not been published yet and a more detailed discussion is beyond the scope of this paper.

\section{THE OMNITREAD CONCEPT}

The OmniTread OT-4 comprises seven segments and six 2-DOF joints, as shown in Figure 3. The segment in the center is called "center segment" or, to emphasize its function, "motor segment" because it houses the single drive motor. All other segments are called "actuation segments" because they house, among others, the control components for the pneumatic joint actuators. Segments \#1 and \#7 can hold a payload, such as cameras, microphones, and speakers. Segments \#2 and \#6 each hold one miniature aircompressor that produces pneumatic power. Segments \#3 and \#5 hold Li-Polymer batteries. Table I lists some of the OT-4's key specifications. 
Table I. Specifications for the OmniTread OT-4.

\begin{tabular}{|c|c|}
\hline Structure: & Seven segments, six 2-DOF rotary joints \\
\hline $\begin{array}{l}\text { Dimensions: } \\
\mathrm{L} \times \mathrm{W} \times \mathrm{H} \\
\text { Weight } \\
\text { Motor Seg. length } \\
\text { Actuator Seg. length } \\
\text { Joint length }\end{array}$ & $\begin{array}{l}94 \mathrm{~cm}(37 \mathrm{in} .) \times 8.2 \mathrm{~cm}(3.2 \mathrm{in} .) \times 8.2 \mathrm{~cm}(3.2 \mathrm{in} .) \\
4.0 \mathrm{Kg}(9.0 \mathrm{lbs}) \\
10.9 \mathrm{~cm}(4.3 \mathrm{in} .) \\
10.3 \mathrm{~cm}(4.0 \mathrm{in} .) \\
3.6 \mathrm{~cm}(1.4 \mathrm{in} .)\end{array}$ \\
\hline $\begin{array}{l}\text { Performance } \\
\text { Diameter } \\
\text { Lifting power } \\
\text { Flexibility } \\
\text { Radius when bent } \\
\text { tightly } \\
\text { Speed }\end{array}$ & $\begin{array}{l}\text { Can pass through a } 10-\mathrm{cm}(4.0 \text { in. }) \text { hole } \\
\text { When stretched out horizontally, OT- } 4 \text { can lift three segments off the ground. } \\
\text { Joints bend at least } \pm 33^{\circ} \text { in any direction but } \pm 41^{\circ} \text { in principal directions. } \\
\text { Outside: } 22.9 \mathrm{~cm}(9 \mathrm{in} .) \\
\text { Inside: } 15.2 \mathrm{~cm}(6 \mathrm{in} .) \\
15 \mathrm{~cm} / \mathrm{sec}(6 \mathrm{in} . / \mathrm{s})\end{array}$ \\
\hline Control & $\begin{array}{l}\text { Off-board PC, connected through wireless data link. Full proportional control } \\
\text { over angular position of joints, stiffness, and forward/backward drive speed. } \\
\text { Currently, three operators are needed to operate six joysticks for the six 2-DOF } \\
\text { joints. }\end{array}$ \\
\hline \multicolumn{2}{|c|}{$\begin{array}{l}\text { Design features that enable } \\
\text { tetherless operation }\end{array}$} \\
\hline $\begin{array}{l}\text { Pneumatic power } \\
\text { Electric power }\end{array}$ & $\begin{array}{l}\text { Obtained from two onboard mini-compressors. } \\
\text { Obtained from onboard batteries. Sufficient for up to } 75 \mathrm{~min} \text { of operation. }\end{array}$ \\
\hline Micro-clutches & $\begin{array}{l}\text { Any track can be individually engaged or disengaged under computer } \\
\text { control, resulting in significant power savings. }\end{array}$ \\
\hline Wireless control & $\begin{array}{l}\text { Wireless system for sending commands and receiving sensor and telemetry } \\
\text { data. }\end{array}$ \\
\hline
\end{tabular}

The OT- 8 and OT- 4 share these mostly unique features:

1. Tracks-all-around each segment. This design aims at maximizing the coverage of the whole robot body with moving tracks. This feature is tremendously important, since the long, slender body of a serpentine robot rolls over easily in difficult terrain that may not allow the robot to upright itself immediately.

2. The 2-DOF joints are actuated by pneumatic bellows, which produce sufficient torque to lift the three leading or trailing segments up and over obstacles. More importantly, pneumatic bellows provide natural compliance with the terrain. This assures optimal traction on most terrains.

3. A single electric drive motor in the center segment provides rotary power to each segment through a so-called "drive shaft spine" that runs through the whole length of the robot.
We believe this design to be more weight and power efficient than individual motors in each segment. The penalty with this design is a limit to the range of motion (on the order of $\left.40^{\circ}\right)$ and inefficiency when articulating the joints.

In the remainder of this section we discuss features 1 and 2 in more detail. Feature 3 is straightforward and does not warrant an in-depth explanation.

\subsection{Track-all-around}

One doctrine in the design of all OmniTread models is the maximal coverage of all sides of the robot with moving tracks. This doctrine is based on two reasons:

1. Serpentine robots inevitably roll over when traveling over rugged terrain. Since terrain conditions may not allow the robot to upright 
itself immediately, only coverage of all sides with propulsion elements can assure continuation of the mission after a rollover.

2. Any contact between an environment feature and a robot's inert (i.e., not propelling) surface impedes motion or entirely stops the robot (i.e., the robot gets "stuck"). In contrast, any contact between an environmental feature and a propulsion surface produces motion. To express this relation quantitatively, we define the term "Propulsion Ratio, ${ }^{1 "} P_{r} . P_{r}$ is measured as the surface area that provides propulsion, $A_{p}$, divided by the total surface area, $A_{\text {all }}=A_{p}+A_{i}$

$$
P_{r}=A_{p} / A_{\text {all }}
$$

where $A_{i}$ is the inert surface area of the body. To further clarify, $A_{p}$ is the sum of all surface areas that could provide propulsion if in contact with the environment, while $A_{i}$ is the sum of all surface areas that could not.

$P_{r}$ is not only a function of the robot's geometry, but also of the application domain. For example, on flat and hard terrain, $P_{r}$ for a conventional automobile is 1.0 since only the wheels can be in contact with the terrain. That is because in a car no inert area of the periphery could possibly be in contact with the ground, that is, $A_{i}=0$. However, on soft terrain the wheels sink into the ground and on rugged terrain obstacles protrude out of the ground, resulting in potential contact between the ground and portions of the inert body periphery. In this case the propulsion ratio $P_{r}$ is undesirably low.

In practice, serpentine robots with a low propulsion ratio get stuck very easily when trying to move

\footnotetext{
${ }^{1}$ In an earlier paper, John Blitch, former Program Director of the DARPA TMR program and currently Director of the Alliance for Robot Assisted Crisis Assessment and Response, developed the notion of "Traction Fraction"[Blitch (2003)]. The Traction Fraction concept is very similar to the "Propulsion Ratio" concept described here, although both concepts were developed independently. Our first formal formulation of the "Propulsion Ratio" concept was included in a report to our sponsors at the U.S. Department of Energy, in Sept. 2002-see http://www.engin.umich.edu/research/mrl/urpr/Reports/ 2002-Monthly-09.pdf.

${ }^{1}$ We consider the significant similarity between the two independently conceived concepts as support for-but not proof of - their validity.
}

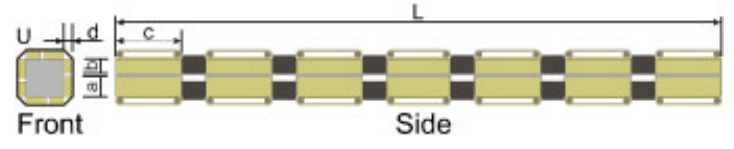

Figure 4. Geometric properties for calculating the propulsion ratio of the OT-4.

over rugged terrain. In order to increase the propulsion area $A_{p}$ and thus the propulsion ratio $P_{r}$, we cover all sides of the OmniTread with extra-wide tracks, as is also advised by Blitch (2003). We also took extensive measures to reduce the space (and, thus, the inert area $A_{i}$ ) between the segments. Environments, in which robots with high propulsion ratios excel, are dense underbrush, rubble, and rocks. In these environments contact can occur anywhere, and robots that have propulsion surfaces only on the bottom are always at risk of being stalled due to excessive, nonpropelling contact. Because of the irregular surfaces of serpentine robots, one can usually not measure $P_{r}$ accurately. Rather, $P_{r}$ is an estimate that is useful for comparing two robot designs.

To illustrate the calculation of the propulsion ratio, we defined the relevant geometric properties of the OT-4 as shown in Figure 4. We simplify the computation of the total surface area, $A_{\text {all }}$, by assuming that $A_{\text {all }}$ is the surface area of a virtual skin that is tightly stretched around the OT-4's body:

$$
A_{\text {all }}=U L+2 A_{e}
$$

where $U$ is the circumference of the virtual skin around a segment, shown as the thick black line in the front view in Figure 4; $L$ is the length of the OT-4, and $A_{e}$ is the face area, that is, the area circumscribed by $U$.

Next, we compute the propulsion area, $A_{p}$, which is made up of the areas of the tracks' plan view orthographic projection areas on the ground (i.e., the area of the shadow under a track if the track was illuminated by parallel light beams from a light source above) as well as the area of the tracks that is visible when looking at the robot straight from the front or the back.

$$
A_{p}=n c(a+b)+2 \cdot 4 d(a+b)
$$

where $n$ is the total number of split tracks $(n$ $=7$ segments $\times 4$ split tracks $=28), a, b$ are the widths 
of each of the two parts of the split tracks (they have different widths), $c$ is the length of a track's projection area on the ground. c includes a projection of one half of the part of the track that is curved around the track sprocket, and $d$ is the height of the track (i.e., diameter of the track sprocket plus twice the thickness of the track).

After substituting the actual dimensions of the OT-4 into Eq. (3) we compute the propulsion ratio for the OT- 4 as $P_{r}=A_{p} / A_{\text {all }}=151,008 \mathrm{~mm}^{2} /$ $295,050 \mathrm{~mm}^{2}=0.51$.

The propulsion ratio for our earlier OmniTread OT- 8 , calculated in the exact same fashion, is about 0.40 .

\subsection{Pneumatic Joint Actuation}

The foremost reason for actuating joints pneumatically is the natural and controllable compliance afforded by this method. Natural compliance is of critical importance, since propulsion depends on optimal traction between propelling surfaces and arbitrarily shaped terrain features. On rugged terrain, maximal traction is achieved by letting joints go limp, allowing the robot's body to conform compliantly to the terrain. Without natural compliance, extremely complex sensor/actuator control algorithms must produce artificial compliance to emulate joint compliance. The inherent compliance of pneumatic joints is also of benefit when the robot falls on rugged terrain: the large impact forces are absorbed in part by the pneumatic joints.

One major problem with pneumatic joint actuation is the difficulty of controlling the somewhat uncommon pneumatic joint actuators. Many more roboticists are familiar with the control of electric motors than with the control of pneumatic actuators.

In order to address the joint control problem methodically, we spent almost 2 years of dedicated efforts studying this problem and developing a good solution. The result is a unique and recently patented pneumatic joint controller. This controller can simultaneously control both the position (i.e., angular deflection) as well as the stiffness of each 2-DOF joint. Furthermore, our controller is optimized for the preservation of compressed air. This is of particular importance in the OT-4 where the flow rate produced by the two onboard mini-compressors is very limited. When we tested the air consumption of conventional pneumatic position-control circuits with our optimized control system, we found that

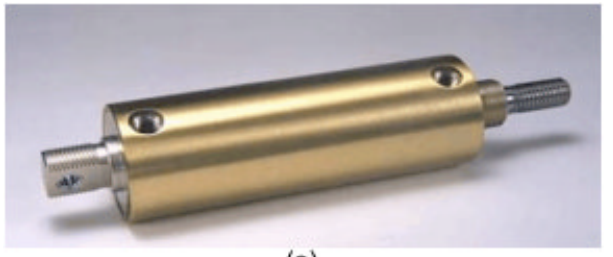

(a)
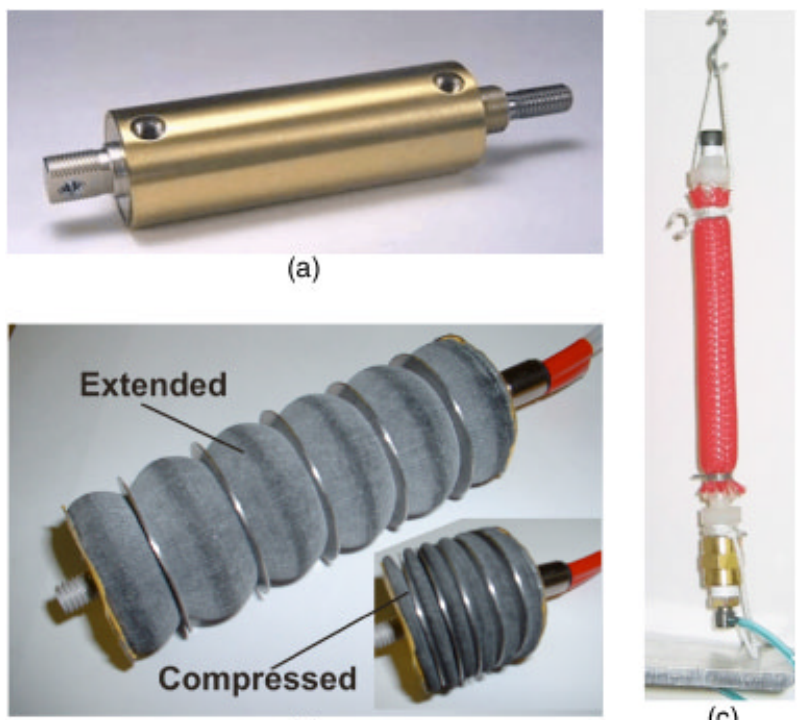

(b)

(c)

Figure 5. Different types of pneumatic actuators. (a) Pneumatic cylinder, (b) pneumatic bellows, and (c) pneumatic muscle ("McKibben muscle").

our system reduced air consumption by a factor of 30. Details on our joint actuation system are provided in our earlier papers [Granosik and Borenstein, 2004, 2005].

There are three well-known types of pneumatic actuators, shown in Figure 5:

- Pneumatic cylinder-This is the most widely used pneumatic actuator. One disadvantage of pneumatic cylinders is their limited strain (i.e., the ratio in length between the fully extended state and the fully retracted state). Because of their design, pneumatic cylinders cannot have a strain greater than 2.0.

- Pneumatic muscle ("McKibben muscle") This actuator comprises an airtight linear, surrounded by a mesh. When the linear is inflated, it balloons, forcing an increase in the diameter of the mesh. Because of the construction of the mesh from diagonally woven fibers, the increase in diameter forces a decrease in length. Thus, inflation of this actuator results in axial contraction. While very forceful, the strain of pneumatic muscles is limited to about 1.3.

- Pneumatic bellows-This actuator is made of an elastic, airtight tube, usually rubber. The 


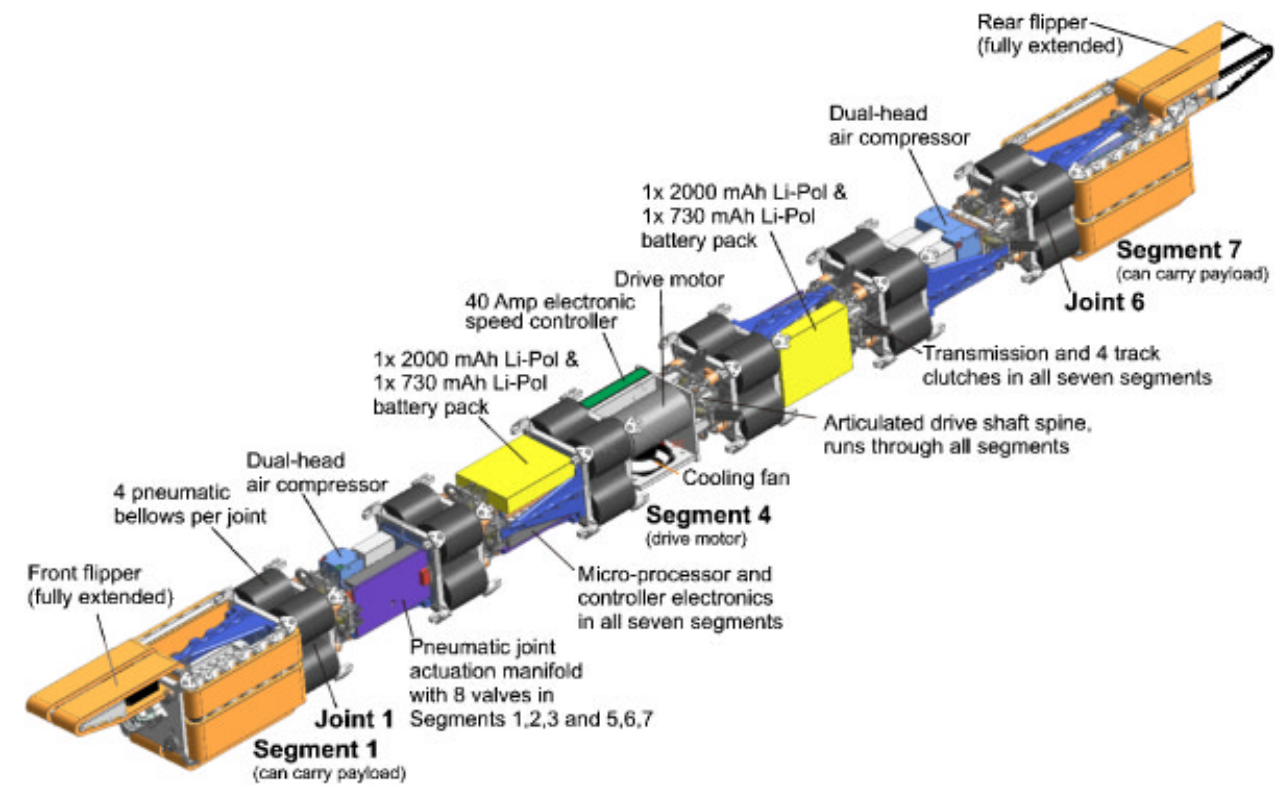

Figure 6. CAD view of all segments of the OT-4. All actuation segments have some common and some special components.

wall of the tube is preshaped into characteristic convolutes that make it easy for the tube to expand axially when inflated. The convolutes also help the bellows fold neatly when axially compressed by external forces. Because of their ability to fold into a compact shape, pneumatic bellows typically have a strain of up to 4.0, that is, their expanded length can be up to four times their fully compressed length. The bellows in Figure 5(b) has fiber-reinforced walls, and metal rings around the inner convolutes prevent the bellows from ballooning. If a bellows was allowed to balloon, it would rupture.

In the OmniTread line of serpentine robots, we chose pneumatic bellows as the joint actuators, since their superior strain allows them to fit entirely into the space of the joint, without taking up any of the very limited space inside the segments. The bellows shown in Figure 5(b) are used in the OT-8. The OT-4 has newer, more sophisticated bellows as will be described in Section 3.6.

\section{DESIGN DETAILS}

In this section we discuss individual functional components of the OmniTread OT-4. Some of these fea- tures are common to the OT-4 and our earlier OT-8, while others are unique to the OT-4. Figure 6 reveals the content and function of each segment of the OT-4.

One key design feature of our OmniTread robots is the placement of a single drive motor in the center segment (see Figure 7). With the motor taking up space only in one segment, all other segments have space for a manifold, valves, and electronic control

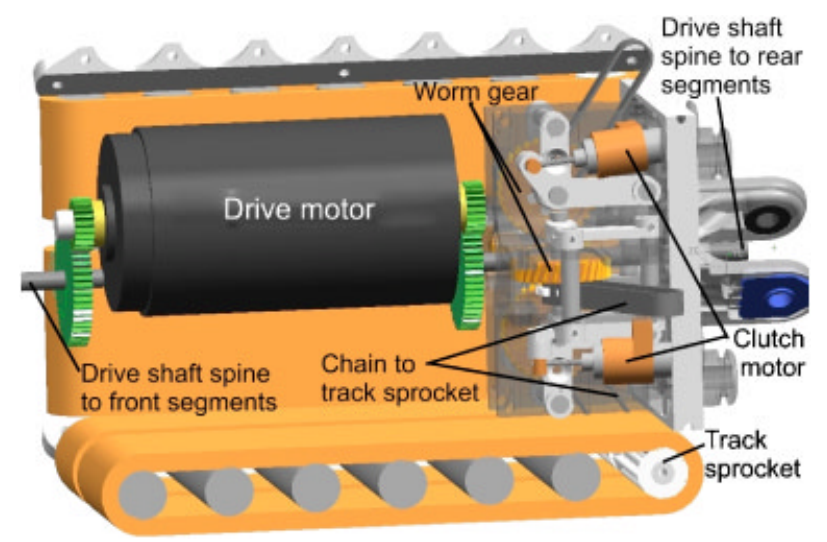

Figure 7. $\mathrm{CAD}$ drawing of the single drive motor in the center segment, along with its reduction gearing and gear box. 


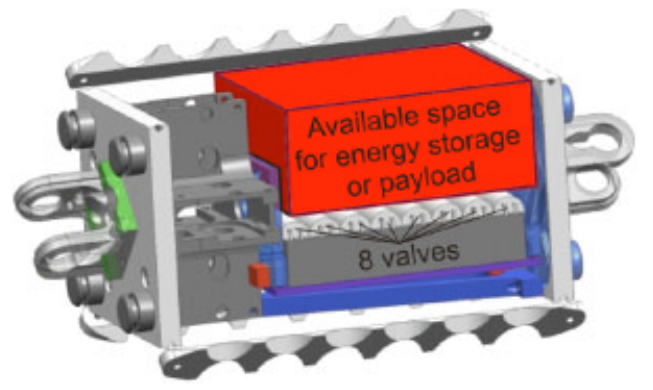

Figure 8. All six nonmotor segments, called "actuation segments," have this space allocation.

boards. In addition, there is a rather large, roughly box-shaped space (shown in Figure 8) that is available for energy storage or for a payload.

\subsection{Drive Train}

The single electric motor in the motor segment powers the so-called "drive shaft spine" that runs the length of the robot. The drive shaft spine comprises seven rigid shafts that are connected by six universal joints. The universal joints are concentrically located within the gimbal joints that link the segments.

On each shaft segment is a worm. Four worm gears feed off that worm on the drive shaft as shown in Figure 9. Each worm gear drives a chain that drives the track sprocket. The drive shaft is sup-

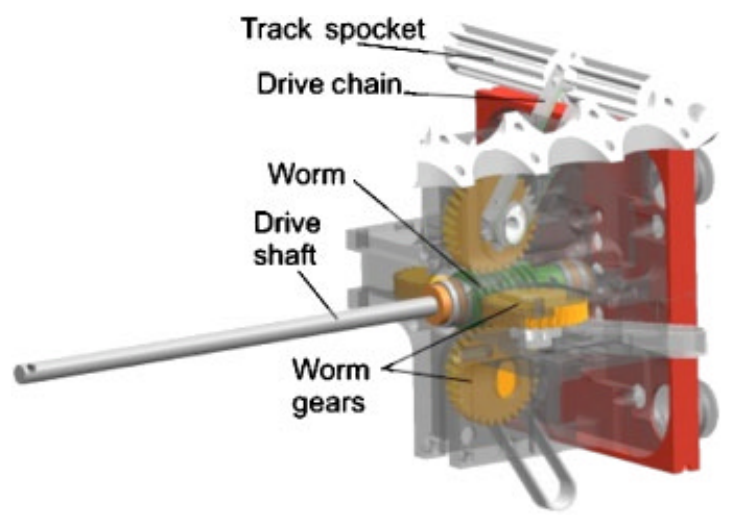

Figure 9. The track sprocket (white) is driven by the driveshaft via a worm (green), worm gear (orange), and chain drive (gray). Micro-clutches, discussed in Section 4.2 , are intentionally omitted from this drawing.

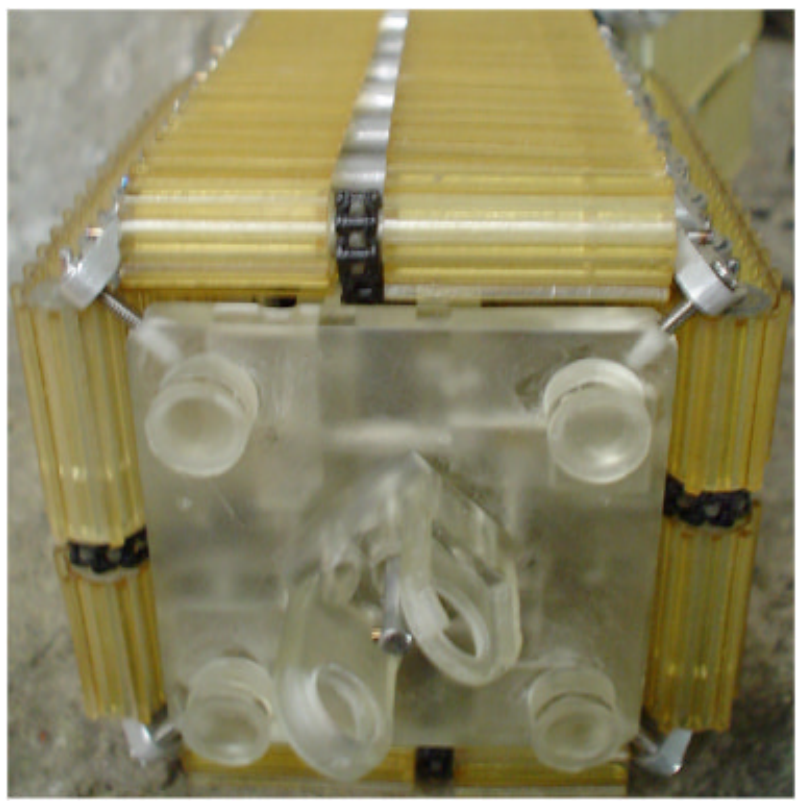

Figure 10. Front view of the OT-4. The extra-wide track areas add stability and reduce the chance of roll-overs.

ported by two ball bearings on each end of the gearbox to retain good tolerances within the gearbox. The other end of the drive shaft is floating and only supported by the universal joint. Not constraining the shaft at three points prevents the driveshaft from flexing too much, if the structure of the segment warps under high loads.

\subsection{Tracks}

To simplify the gearbox, the chain is run off a sprocket mounted directly on the side of the worm gear. The chain drive is therefore off-center with respect to the driveshaft and the two "half"-tracks per side are therefore not of equal width (see Figure 10). We mold the tracks in-house from a silicon mold, which is made from a Stereolithographic (SLA) rapid prototype, based on our CAD model.

The grousers have twice the pitch of the track teeth to better engage features of the obstacle being scaled. Keeping the grouser pitch a function of the tooth pitch reduces the stiffness of the track, as most of the flexibility of the track comes from the thin area between the teeth.

In order to reduce the chance of rollovers, it is desirable to make the tracks as wide as possible. 
Table II. Maximal and minimal torques in the OT-4 drive train.

\begin{tabular}{|c|c|c|c|c|c|}
\hline Situation & $\begin{array}{l}\text { Tangential } \\
\text { load on } \\
\text { track }(\mathrm{N})\end{array}$ & $\begin{array}{c}\text { Torque at } \\
\text { track } \\
\text { sprocket }(\mathrm{Nm})\end{array}$ & $\begin{array}{c}\text { Tension } \\
\text { in chain } \\
(\mathrm{N})\end{array}$ & $\begin{array}{l}\text { Torque at } \\
\text { worm gear } \\
(\mathrm{Nm})\end{array}$ & $\begin{array}{l}\text { Torque at } \\
\text { drive shaft } \\
\text { (Nm) }\end{array}$ \\
\hline Driving single track freely ${ }^{\mathrm{a}}$ & 0.0 & 0.10 & 22 & 0.09 & 0.012 \\
\hline Climbing vertically inside a pipe ${ }^{b, c}$ & 20 & 0.43 & 94 & 0.38 & 0.051 \\
\hline Breaking a chain (minimum) & 37 & 0.64 & 141 & 0.57 & 0.076 \\
\hline Breaking a chain (average) & 41 & 0.66 & 146 & 0.59 & 0.078 \\
\hline
\end{tabular}

${ }^{\mathrm{a}}$ Track spins in the air.

${ }^{b}$ This condition applies only to one track of the center segment. The tracks on the distal segments that touch the other side of the inside wall of the pipe each carry about $25 \%$ of the robot's weight.

'Since the track is pressed hard against the inside wall of the pipe, the friction in the track sprocket bearing is very high and therefore the torque required to turn the track sprocket is higher than the calculated value based on the track sprocket diameter.

This is especially important considering the large deflection of the center of gravity that we can impose on the robot by raising three segments in the air.

On the other hand, our design goal was to let the OT-4 pass through a 4 in. diameter hole-clearly a requirement that conflicted with making the tracks as wide as possible. To meet both goals, we had to minimize the sprocket diameter, as is evident from Figure 10. The disadvantages of small sprockets are (1) greater roll resistance, (2) reduced ability to accommodate sand and small particles, and (3) reduced ability to transfer torque between the sprocket and the track. In order to transfer more torque, the tooth profile was kept similar to that of a timing belt, i.e., we maximized the number of engaging teeth.

\subsection{Torque Analysis}

In this section we present a brief analysis of torques in the drive system. We consider here only the two extreme cases: (1) No load on a track, that is, a track spinning freely in the air, and (2) maximal nominal load on a track. The latter case occurs when the OT-4 travels upward inside vertical pipes of six or more inches inside diameter (see details on this experiment in Section 5.6). In this case the robot assumes a letter " $\mathrm{C}$ " shape, so that its distal segments press against one side inside the pipe, and the center segment presses against the other side. In this situation the load on the center segment's track that touches the inside wall of the pipe is about one half of the robot's total weight. This is the largest legitimate load to be expected under normal conditions, and we denote it $F_{\max }$. Of course, even larger loads may develop if a track becomes jammed. Such overloads can cause part breakages. Table II lists some of the torques and forces that prevail in the drive system under minimal and maximal load conditions.

\subsubsection{Shear Pins}

Especially at risk for breakage is the weakest link in the drive train: the chain. We cannot increase the strength of the chain because we are already using the strongest chain that can be custom-fabricated for the required dimensions.

To prevent damage to the drive system and especially the chain, we developed shear pins that are located in the track sprockets. The shear pins are designed to break when loads on the track exceed $F_{\max }$, but before the chains break. Thus, and according to Table II, the shear pins in the track sprocket are designed to break at a torque of about $0.5 \mathrm{Nm}$.

\subsubsection{Drive Motor}

The single drive motor in the center segment is Model 2232-012 SR made by Faulhaber. The performance chart (torque, speed, and power) for this motor is shown in Figure 11. Under normal driving conditions, even when all 28 track clutches are engaged, the total torque requirement at the drive shaft (see Table II) is $28 \times 0.012=0.34 \mathrm{Nm}$. This is well within the motor's performance range. Under the maximal load condition (driving upward inside a vertical pipe) and even when all 28 tracks are engaged, the torque on the motor increases only to about 25 $\times 0.012+3 \times 0.051=0.45 \mathrm{Nm}$. In this calculation we used data from Table II again, and we made the overly conservative assumption that three tracks 


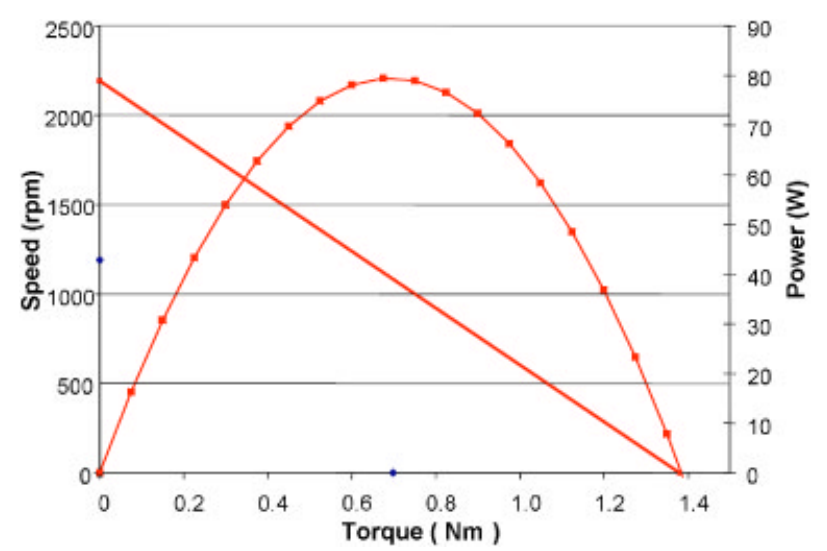

Figure 11. Torque, speed, and power chart for the Faulhaber Model 2232-012 SR motor.

were each bearing half the robot's weight. Even under these exaggerated conditions, the motor is easily capable of producing the required torque. Indeed, at stalling speed the motor has enough torque to break $1.4 / 0.078=17$ chains simultaneously. Of course, the shear pins would break before the chains do.

In practice, we found that despite the protection of the chains by shear pins, drive train parts in the robot can fail. These failures are typically the result of multiple tracks in distal segments being overloaded, but not overloaded enough to break the shear pins. Under these conditions the universal joints that transfer torque between the segments of the drive shaft spine can break. Such overload conditions in multiple tracks can develop when the robot ingests sand or twigs into the drive system while frequently rolling over. A possible solution for this problem is the automatic disengagement of the clutches of the affected tracks, based on force measurements in the chain. This can be done, in principle, by measuring the slack of the chain during operation. However, we have not implemented this solution, yet.

\subsection{Chassis Design}

Due to the small size of the OT-4, significant efforts had to be made to organize the internal components for space efficiency and accessibility. Cables and pneumatic lines are routed with these goals in mind. For example, the electronic circuit board on each segment has a connector on both ends, with the

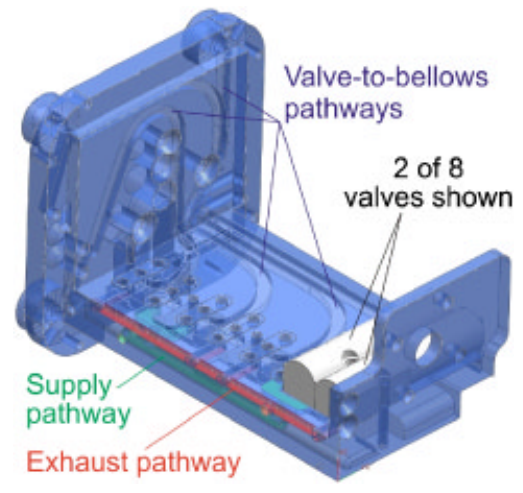

Figure 12. Manifold with two of the eight valves (white) mounted. Exhaust and supply pathways from and to the bellows are shown in darker colors.

wires coming from the neighboring segments plugging into the closer side. This design eliminated the need for wire runs all the way through the segment. Similarly, we integrated most of the pneumatic pathways into the chassis, while using pneumatic hoses only for exchanging air between segments. This was possible thanks to SLA rapid prototyping techniques, which build the parts in layers and allow for such internal features. The chassis with integrated manifold and "etched-in" pneumatic pathways is shown in Figure 12.

SLA rapid prototyping allowed us to create very complex, and otherwise difficult-to-machine, structures. The SLA technique also allowed us to design parts for ease of assembly, maintenance, and space savings. However, SLA resins tend to warp with time, which is why they are normally used for prototyping only. In our early OT-4 prototypes, components that were under constant load would creep with time and would cause problems, especially in the case of the seal between the valves and the manifold. Aluminum reinforcements were therefore added to the endwalls, joints, and manifold at key points where creep and deformation during load were becoming an issue. Figure 13 shows these reinforcements (in dark shades). Specifically, the endwalls were reinforced with a thin aluminum shell and the manifold was reinforced with an aluminum bar screwed on at both ends. The result was a much stiffer segment at a minor (2.5\%) weight penalty. 

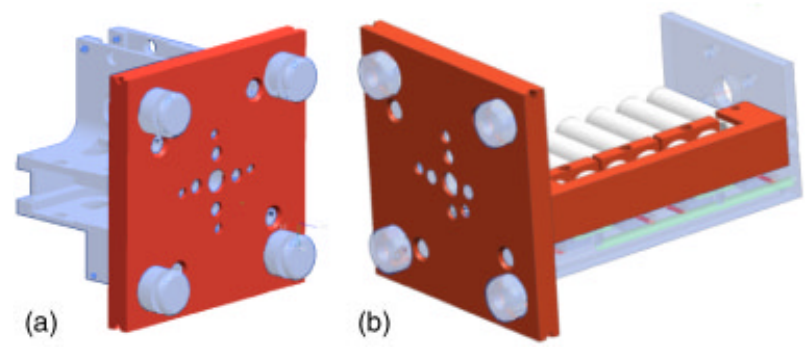

Figure 13. Aluminum-reinforced SLA parts. (a) Rear of segment; (b) front of segment.

\subsection{Joints}

Between any two segments are two concentric universal joints that are referred to as the "outer" and "inner" universal joint. The outer universal joint connects the two adjacent segments. It is made of two forks and a ball bearing-mounted gimbal connecting the two forks, as shown in Figure 14. The inner universal joint (not shown) connects adjacent segments of the drive shaft spine and is concentrically located inside the gimbal. All components of the outer universal joint are made from aluminum and each fork is screwed onto the adjacent segment endwalls. Two Hall-effect angle sensors are mounted on arms of the outer universal joint, as shown in Figure 14. These sensors provide position feedback for the control of the two joint angles.

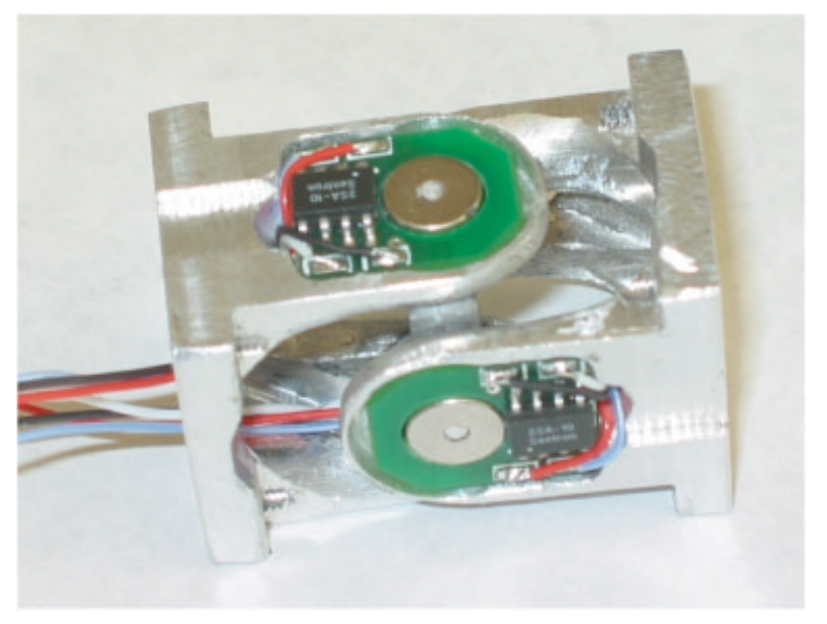

Figure 14. Outer universal joint with two Hall-effect angle sensors, one for each axis of rotation.
The joint can be actuated at least $33^{\circ}$ in any direction and up to $41^{\circ}$ in the four principal directions (up, down, and side to side). Wiring and pneumatic lines between the segments pass through four holes at the corners of the gimbal and the bases of the forks.

Each joint is orientated with respect to the other in a way so as to compensate for gimbal error, the angular "twisting" deviation that occurs between the two ends of a universal joint as it is articulated. Without this, three fully articulated joints would lead to each progressive segment being "twisted" about the drive spine axis, leading to instability and making traversing obstacles difficult.

\subsection{Pneumatic Bellows}

Pneumatic bellows develop axial force according to

$$
F=P A
$$

where $P$ is the pressure of the compressed air and $A$ is the area of the bellows surface that is normal to the axial direction, that is, the area of the cross section. One problem with Eq. (4) is the difficulty in determining exactly what the area $A$ is. For example, in the bellows shown in Figure 5(b) (back in Section $2.2)$, the convolutes change the diameter and thus the area of the cross section along the bellows. Of particular concern is the minimal cross section area, $A_{\text {min }}$, which corresponds to the inner whorl of the convolutes. In theory, for a given pressure $P$, the axial force that the bellows can apply is limited by the cross section area of the inner whorls, $A_{\min }$ (in practice, though, $F$ is larger than $P A_{\min }$, likely because in a pressurized bellows the neoprene liner acts more like a rigid cylinder wall and thus contributes to $F$ ). Yet, the volume of space that the bellows requires is determined by the diameter of its outer whorls. In the relatively large OT- 8 , the ratio between the inner and outer diameters of the whorls (we call this ratio "bellows efficiency") is fairly close to 1.0. However, in the smaller bellows of the OT-4, the bellows efficiency is much smaller than 1.0. In many conventional bellows the diameter of the inner whorl increases when inflated, thereby improving that bellows' efficiency. However, our OT- 8 bellows design uses a metal ring around the inner whorls to prevent the bellows from ballooning. At the same time, these rings prevent the inner whorls from 


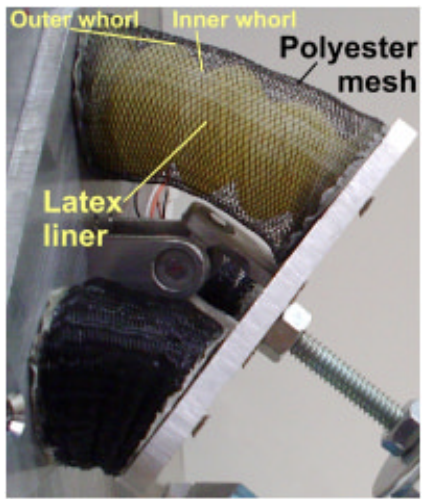

Figure 15. OT-4 bellows comprising a liner and a mesh. We chose yellow latex linear material for this photograph because it contrasts better with the black mesh. However, the actual OT-4 bellows have neoprene (black) liners.

growing in diameter, thereby keeping the bellows efficiency low.

To overcome this problem in the small-sized OT-4 bellows, we abandoned the metal rings altogether. Instead, we encased the OT-4 bellows in a tubular polyester mesh. To distinguish between these parts, we call the airtight, elastic part of the bellows "liner," and the outer part "mesh."

The new two-part bellows of the OT-4, shown in Figure 15, has the significant advantage of allowing the diameter of the inner whorl to grow when pressurized, until the inner whorl is practically flush with the mesh (see Figure 16). The result is a bellows that has an efficiency of close to 1.0, when pressurized.

There is, however, one problem with all bellows designs: When the bellows extends beyond the natural length of the liner, the axial extension force $F$ $=P A$ has to work against the elasticity of the liner. Similarly, when a bellows in a joint is compressed beyond a certain limit (e.g., because the bellows on the opposite site is expanding), its liner and mesh develop elastic forces that resist further compression with increasing force.

As a result of these effects, the moment produced by the bellows when installed inside a joint is neither constant nor depending only on the applied pressure differential. Rather, the produced moment is a nonlinear function of the joint's momentary angle. For extreme joint angles, the moment produced by the joints may be reduced by as much as

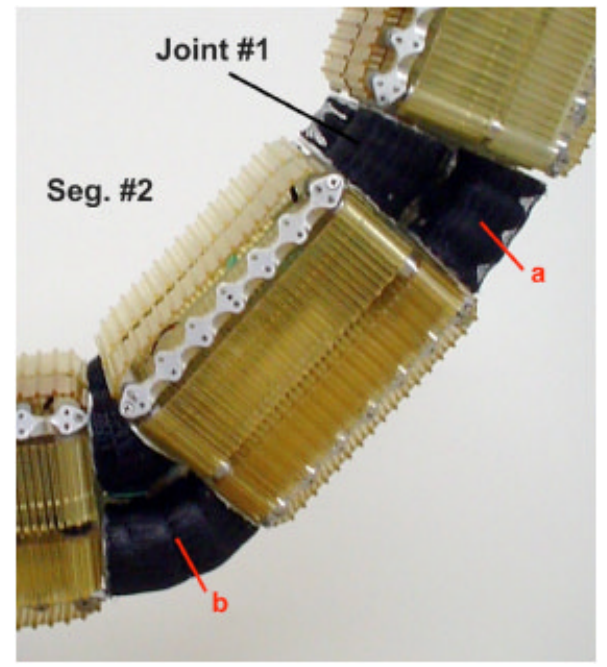

Figure 16. OT-4 bellows at different levels of internal pressure. Bellows "a" in joint \#1 is pressurized to a moderate level. Bellows " $\mathrm{b}$ " is inflated to the maximal pressure of 30 psi.

$80 \%$ compared to the maximal moment that is available when the joint is in its neutral position. Despite these limitations, the bellows in the OT-4 are powerful enough to lift three segments even at nearmaximal joint angles.

\section{ADVANCED FEATURES}

Up to this point we discussed mostly features and properties that are common to our older OmniTread OT- 8 and the OT-4. For this reason our earlier papers on the control system and design considerations for the OT-8 (Granosik, Hansen \& Borenstein, 2005) are also applicable to the OT-4 and they are therefore not addressed in this paper. However, the OT-4 has several unique features that are not found in the OT-8. We discuss the most salient of them in this section.

\subsection{Completely Tetherless Operation}

The OT- 8 required three resources to be supplied to the robot through a tether: electric power, compressed air at $80 \mathrm{psi}$, and control signals. In order to make the OT- 4 entirely tetherless, these resources have to be supplied onboard. We discuss here how these resources are provided onboard the OT-4. 


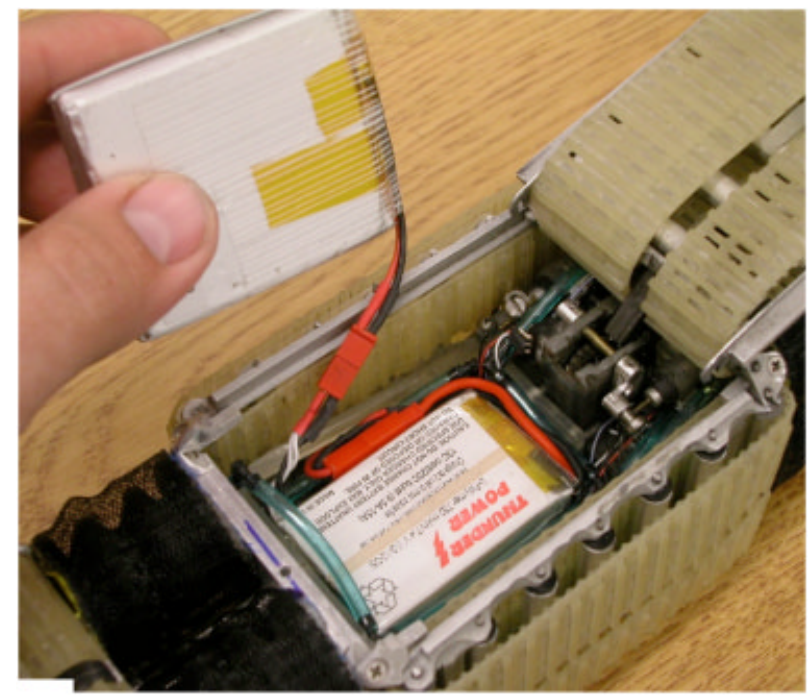

Figure 17. The $730 \mathrm{mAh}$ Li-Pol battery is in its place in segment \#3. The remaining space will be completely filled by the 2000 mAh Li-Pol battery in the engineer's hand. An identical set of batteries is located in segment \#5.

\subsubsection{Electric Power}

The OT-4 has two electric power circuits: a motor power circuit and a control power circuit.

1. The motor power circuit powers the drive motor and the two onboard minicompressors. This power is supplied by two 7.4 V, $2000 \mathrm{mAh}$ Li-Pol batteries, one each stored in segments \#3 and \#5. The two batteries are connected in series to provide $14.8 \mathrm{~V}$ and their total energy storage capacity is 29.6 Whr. These batteries take up a volume of $84 \mathrm{~cm}^{3}$ and weigh $160 \mathrm{~g}$.

2. The control power circuit powers the electronics control boards and pneumatic values, as well as the wireless communication system. This power is supplied by two $7.4 \mathrm{~V}$, $730 \mathrm{mAh}$ Li-Pol batteries, one in segment \#3 and one in segment \#5. The two batteries are connected in parallel to provide $1460 \mathrm{mAh}$ at $7.4 \mathrm{~V}$ and their total energy storage capacity is $10.8 \mathrm{Whr}$. These batteries take up a volume of $34 \mathrm{~cm}^{3}$ and weigh a total of $76 \mathrm{~g}$.

In total, all four onboard batteries store $40.4 \mathrm{Whr}$ of electric energy, occupy a volume of $118 \mathrm{~cm}^{3}$, and weigh $236 \mathrm{~g}$. Figure 17 shows one of the two battery-

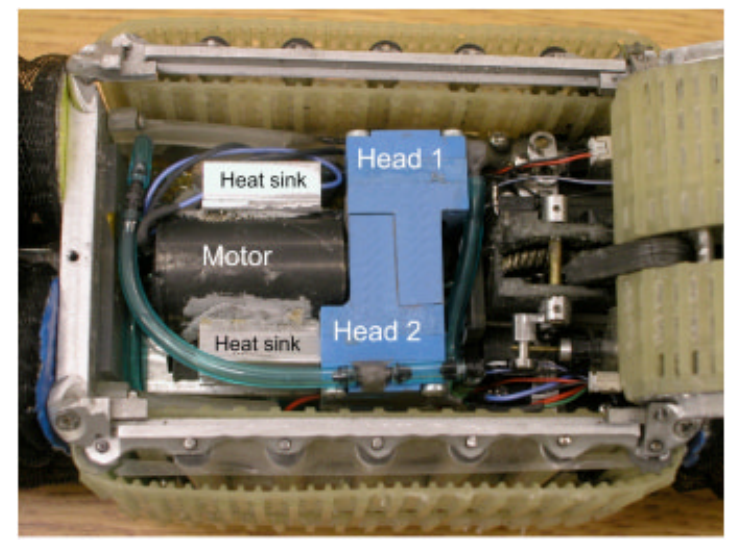

Figure 18. Modified dual-head mini-compressor.

holding segments with its $730 \mathrm{mAh}$ and $2000 \mathrm{mAh}$ batteries.

In an endurance test (driving as far and long as possible on one charge on flat concrete floor) the motor power and the control power batteries lasted roughly the same time. Details on the OT-4's performance in the endurance test will be presented in Section 5.1. On extremely difficult obstacles, where joints are actuated a lot and all tracks are engaged, the motor battery can be depleted in as little as $25 \mathrm{~min}$.

\subsubsection{Pneumatic Power}

Pneumatic power is supplied by two off-the-shelf Hargraves CTS single-head mini-compressors, one each in segments \#2 and \#6 (see Figure 6). In order to increase the flow rate we added a second compressor head in parallel to the existing single head of each of the two compressors. (See Figure 18.) This way, in one revolution of the crankshaft two pistons go through a compression cycle instead of just one. We modified the dual-head mini-compressors further by replacing the stock motor by a more powerful one, the Faulhaber Model 2232012 SR. The Faulhaber motor is coreless, slightly larger than the stock motor, and has a higher power rating. Because of that higher rating, it draws only one third the current of the stock motor, which was somewhat overloaded when running two heads.

In this configuration, which we call "Parallel Mode," the mini-compressors provide about 25 psi in the robot (less when flow rates through the robot 


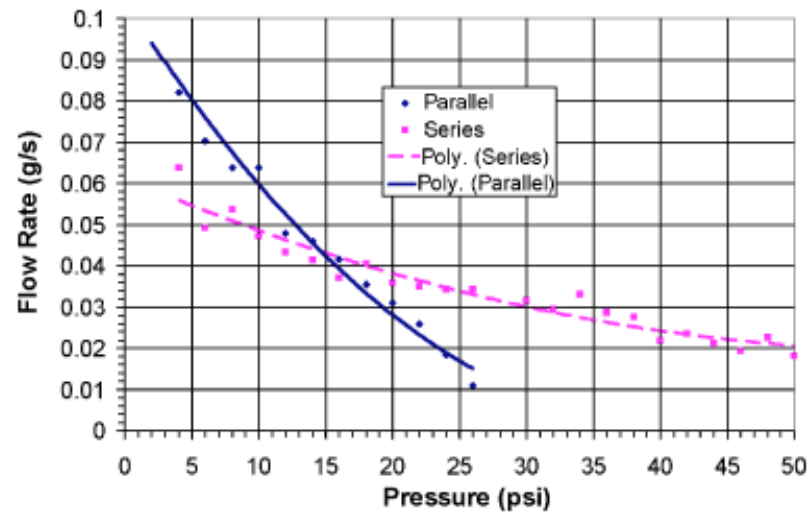

Figure 19. Plot of flow rate versus pressure for the dualpressure compressor working in series (solid line) and parallel (interrupted line) mode. This chart is for one minicompressor, although the OT-4 uses two mini-compressors.

are high). This maximal pressure is sufficient for most ordinary tasks with the OT-4, since its bellows were specifically designed for a much lower operating pressure than that of the OT-8. However, for extreme tasks, such as the vertical climb in largediameter pipes and other tasks with vertical motion requirements, a higher pressure is desirable. That is because higher pressures translate into proportionally greater joint actuation torques. To achieve this higher pressure we connected the two heads of each compressor in series, thereby increasing the effective output pressure of one mini-compressor to $\sim 50$ psi.

Figure 19 compares the pressure and flow rate produced in both compressor modes. For low pressures up to $\sim 15$ psi the flow rate produced in Parallel Mode is greater. Above $15 \mathrm{psi}$, the flow rate in Series Mode is greater. Since we are always interested in the highest possible flow rate at any pressure, it would be desirable to be able to switch between modes, depending on the needed or prevailing pressure.

It is possible to do just that by means of a single solenoid valve and a check-valve, as shown in the pneumatic diagram of Figure 20. In principal, switching between the two modes can be done anytime during operation, without stopping the compressors. However, it is difficult to find a sufficiently small solenoid valve with high enough flow rate to implement this design in the very limited confines of the OT-4. For this reason we currently switch manu-

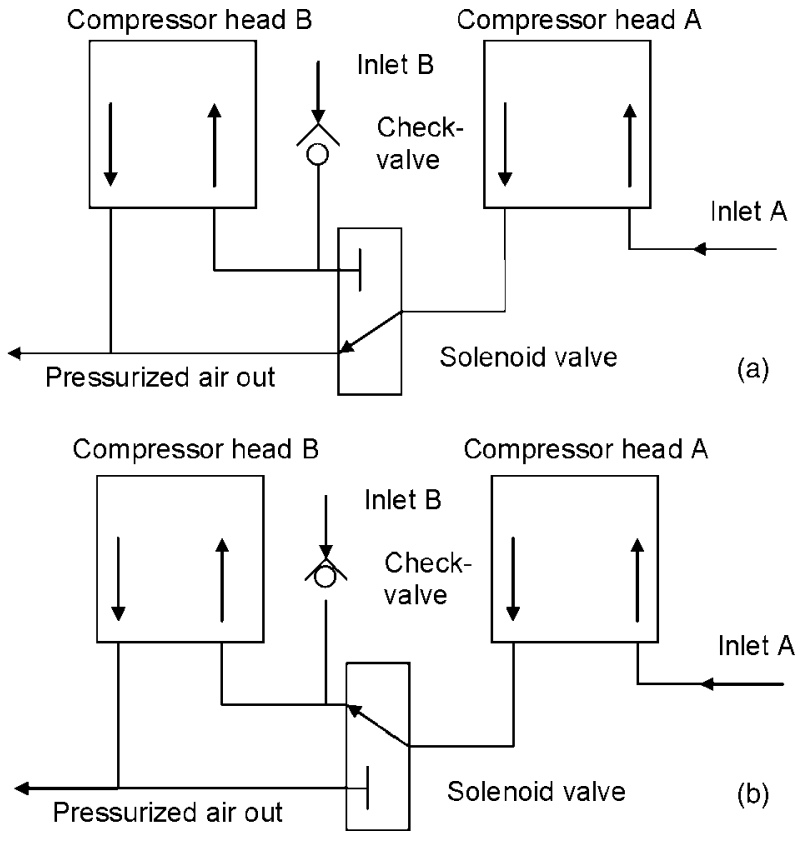

Figure 20. A run-time switchable dual-pressure compressor system. The compressor heads can be switched between (a) Parallel Mode and (b) Series Mode by switching the state of the solenoid valve.

ally between the two modes, prior to a mission and depending on the type of mission. The default setting is Parallel Mode.

\subsubsection{Wireless Communication System}

In the OT-8 the communication of control signals from the joysticks (via a laptop) to the robot and sensor signals from the robot to the off-board laptop were sent through the tether, as shown in Figure 21(a). In the OT-4, we implemented the wireless communication system of Figure 21(b).

Our solution involved removal of the housing and other components from a Lawicel CAN-to-RS232 converter to reduce its volume, and wiring it to a Maxstream Xbee transceiver. Despite the complexity of the multiple-conversions system, we managed to integrate the components into the OT-4's tail segment such that most of the payload space in that segment remained available. Figure 22 shows the on-board components of the wireless communication system.

The range of the system is approximately $20 \mathrm{~m}$ 


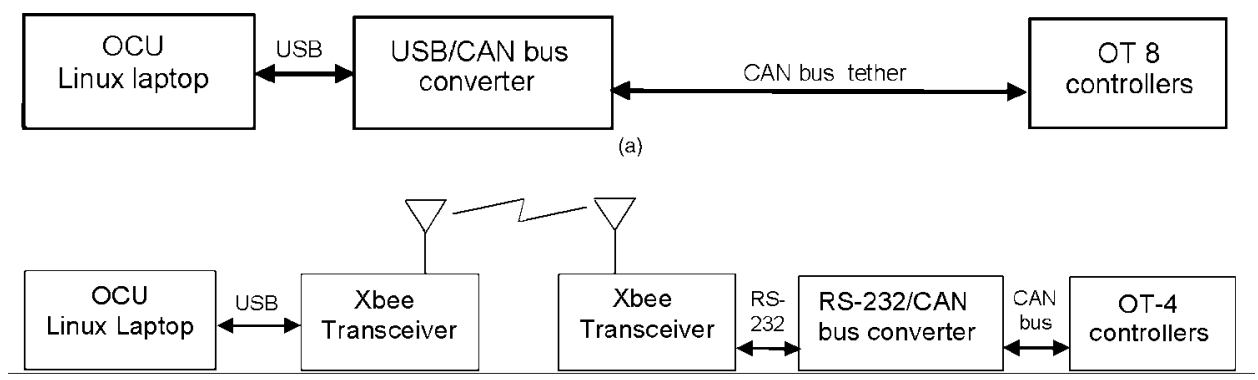

(b)

Figure 21. Control communication system components. (a) Tethered system for the OT-8. (b) Wireless system for the OT-4.

through two walls with no apparent problems. The CAN message throughput of the wireless system is slightly lower than that of the OT-8's tethered system despite similar transmission baud rates $(115.2 \mathrm{~K}$ vs $125 \mathrm{~K}$ ). This is because the messages are transmitted in the wireless system as ASCII strings rather than as binary ones.

\subsection{Electrically Actuated Micro-clutches}

One reason for then OT-4's impressive motor battery run time (75 min on benign terrain) is a unique fea-

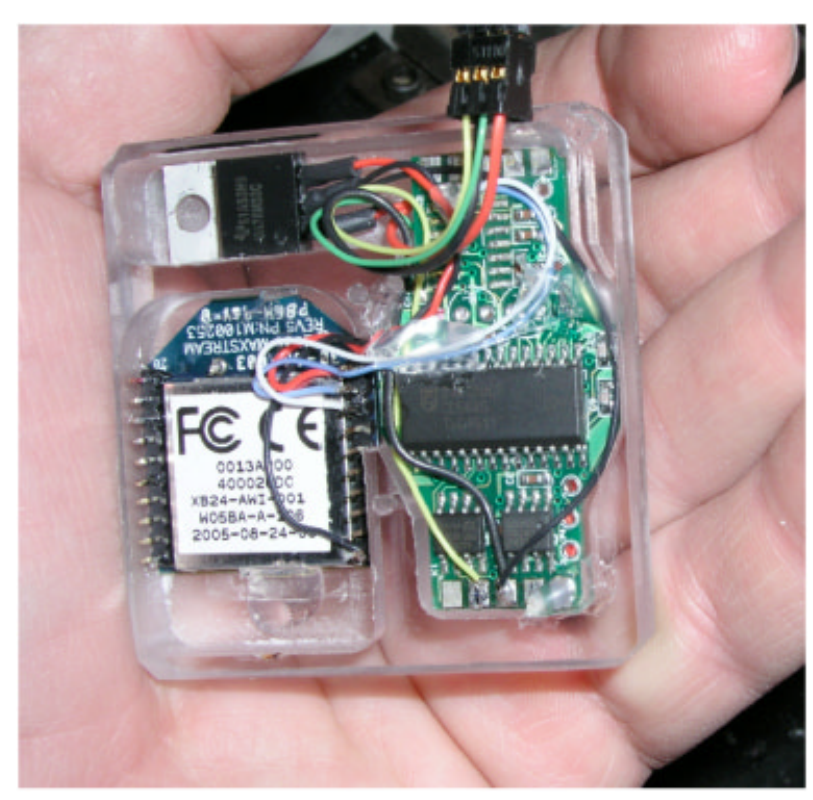

Figure 22. Onboard components of the OT-4's wireless control communication system. ture in the OT-4: all 28 of its tracks can be engaged or disengaged from the drive train individually and under computer control. To motivate the utility of the clutches, let us consider some figures (from Table II in Section 3.3.1). A torque of $T_{f}=0.10 \mathrm{Nm}$ is needed to drive a freely spinning track, that is, a track that is not engaged with any environmental feature. At the other extreme, the largest possible legitimate torque that a track may have to transfer is needed during vertical pipe climbs. During such climbs, one track of the center segment is pressed against the inside wall of the pipe and has to support half the robot's weight. Under this condition the torque required to turn that track is $T_{m}=0.42 \mathrm{Nm}$. Comparing these two extreme torque requirements shows a ratio of $q=T_{m} / T_{f}=4.2$. The significance of this ratio is that driving $\sim 4$ tracks at the lightest possible load (i.e., spinning freely) requires the same amount of torque as driving one track under the largest possible load condition. Since torque is roughly proportional to power consumption, we conclude that idly turning four tracks consumes as much power as driving half the robot's weight vertically. It is thus obvious that not driving an idle track will save a substantial amount of onboard electric power.

In practice we implemented the micro-clutches as shown in Figure 23. To disengage a track, a micromotor moves one link of a four-bar mechanism so that the worm gear is lifted off the worm. Microswitches (not shown here) stop the micro-motor in two stable, self-locking positions. These positions correspond to the worm gear being fully engaged or disengaged from the worm. We designed the fourbar mechanism such that the micro-motor and lead 


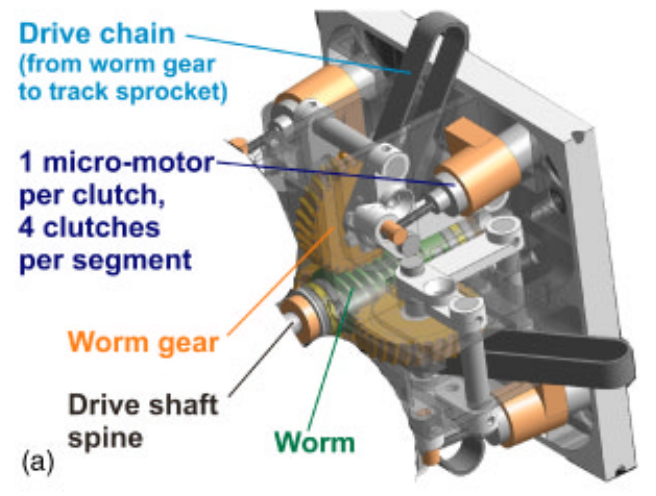

(b)

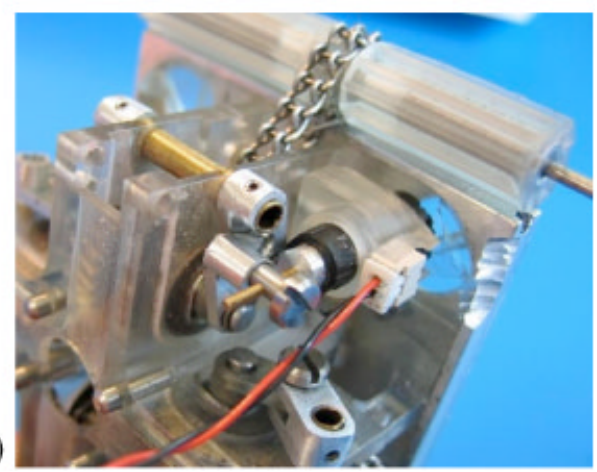

Figure 23. Gear box and micro-clutches. (a) CAD drawing. (b) Photograph.

screw mechanism are subjected only to a very small part of the potentially very large worm/worm-gear separation force.

\subsection{Flipper Tracks}

We equipped the OT-4 with two so-called "flipper tracks" (see Figure 24). These tracks, located in the lead and tail segments, can be "flipped out" or "flipped in" to extend the reach of the OT-4. The extended reach is useful in two maneuvers: (a) to cross gaps and (b) to reach up and over high obstacles. The flipper track uses a small servo embedded in the track tray to extend the flipper $180^{\circ}$ or to retract it. An additional locking actuator locks the track in either position. The servo is slightly wider than the height of the track, resulting in the outward bulge of the track, apparent in Figure 24. Yet, the bulge does not interfere with the robot's ability to pass through a $10 \mathrm{~cm}$ (4 in.) diameter hole.

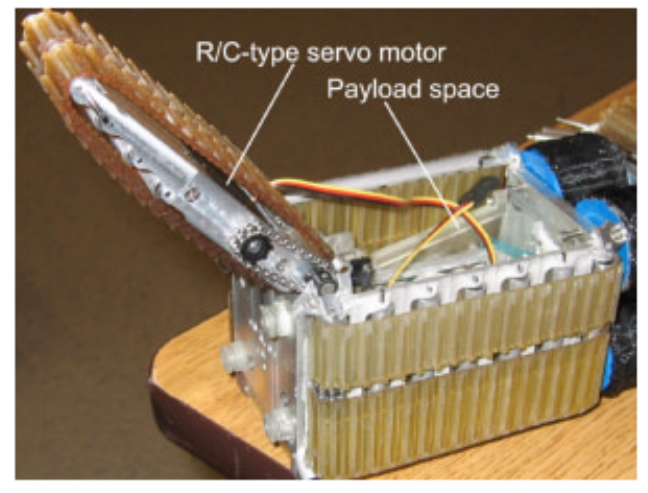

Figure 24. Flipper track during deployment to its fully extended position.

\section{EXPERIMENTAL RESULTS}

In early August 2006, the OmniTread OT-4 was tested for 4 days by an independent and objective third party: the Southwest Research Institute (SwRI). SwRI has developed a variety of test environments for small mobile robots. Each test environment at SwRI has numerous obstacles or other difficulties (collectively called "Challenges") that are designed to test the limits of different types of robots. Many of the photographs shown in this section were taken during testing at SwRI, while others are from testing at our lab at the University of Michigan. All photographs in the remainder of this section were taken during successful traverses of the Challenges shown. In addition to the photographs and text of this section, we refer the reader to our library of high-resolution video clips and photographs of the OT-4 in different environments at http://www.engin.umich.edu/research/ mrl/00MoRob_6.html.

While the maximum speed of the OT-4 is $15 \mathrm{~cm} / \mathrm{s}$, the average speed during obstacle traverses is much slower. That is because most of the Challenges require a great deal of coordination among the three joystick operators (each operator controls the deflection and stiffness of two OT-4 joints-see Figure 25). An additional difficulty is that for many situations the operators had to devise a motion sequence that would allow the robot to traverse the Challenge. This "learn-as-you-go" operation and the required coordination among three operators resulted in many ineffective moves including many unnecessary rollovers, as the OT- 4 center of gravity was raised to an 


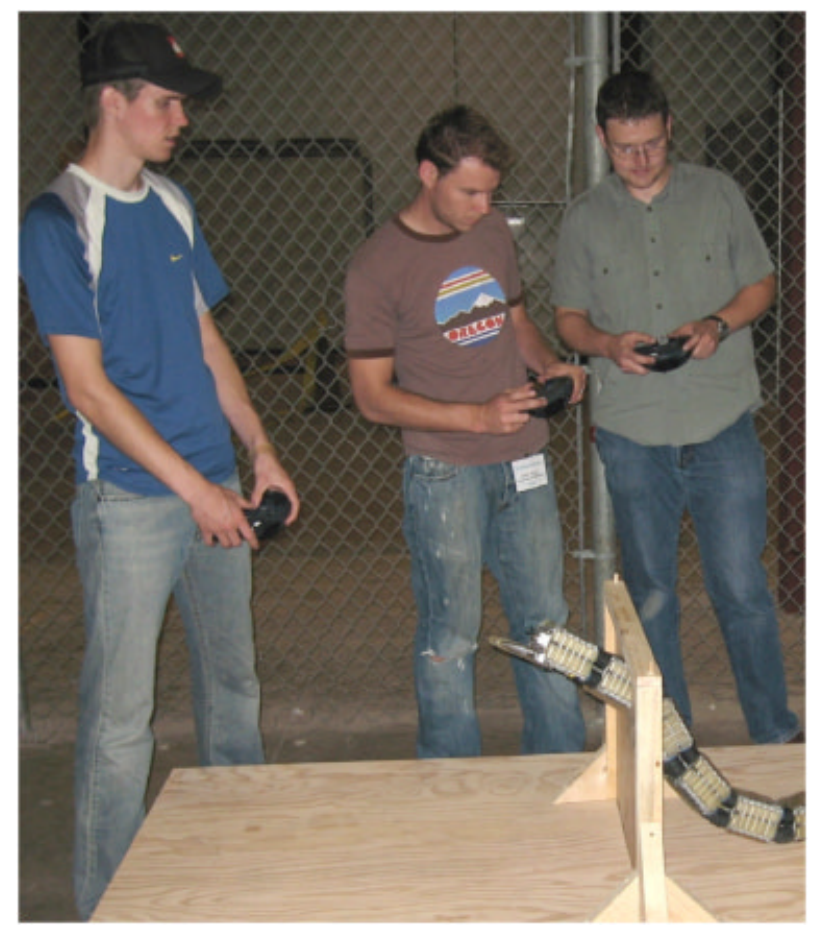

Figure 25. Three operators are needed to control the OT4's six joints.

unstable level. Limited coordination, ineffective moves, and rollovers did not result in failures to traverse the Challenges, but they did result in a substantially lower average speed in most Challenges. Clearly, computer assistance for coordinating the motion and applying smart motion sequences would be of great help in reducing the number of operators and streamlining the motion. However, this paper focuses only on the OmniTread's electro-mechanical system, and not on the very complex computer control problem.

In the remainder of this section, we discuss the OT-4's performance in some of the Challenges.

\subsection{Endurance Test}

The goal of the endurance test was to establish the maximal drive time on a single set of batteries. During the test the robot drove along a square shaped path that required only minimal joint actuation for steering (see Figure 26). At the onset of the test, the robot assumed a specific pose, with which it touched the smooth concrete floor with only three segments,

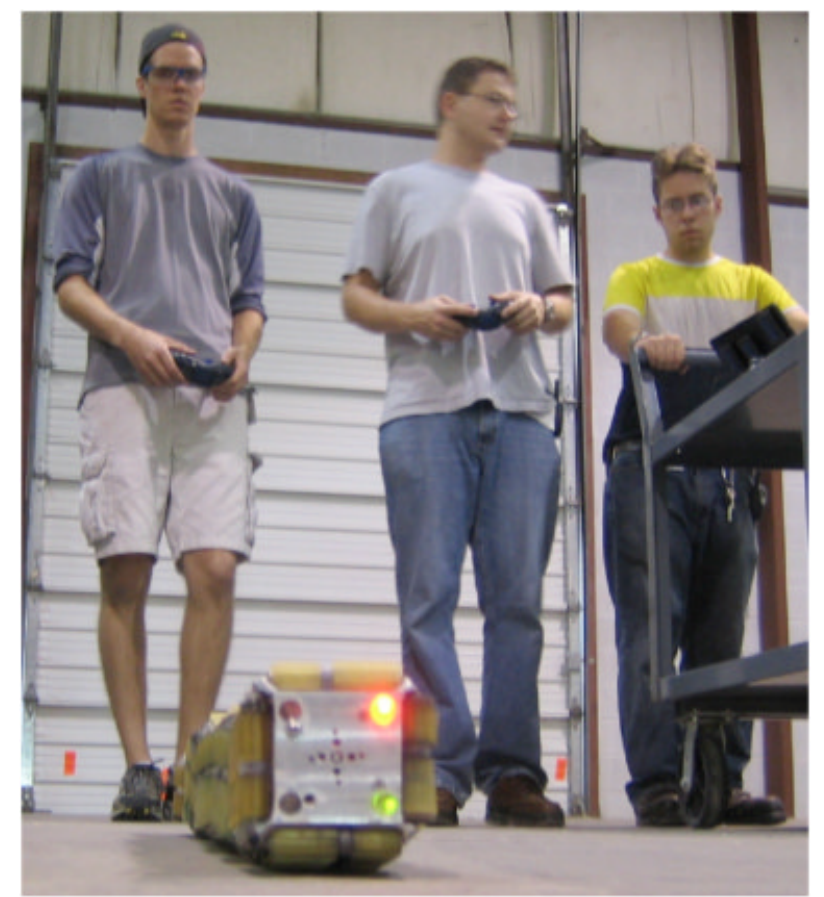

Figure 26. During the endurance test the OT-4 drove along a square shaped path that required minimal joint actuation.

while the other segments were slightly raised off the ground. Using the micro-clutches, we disengaged all tracks except for the bottom tracks of the segments that touched the floor. In this configuration we could still steer the robot around the corners of the squareshaped path, but energy losses from driving tracks idly were minimized. In the test, the OT-4 drove for $75 \mathrm{~min}$ at an average speed of $12 \mathrm{~cm} / \mathrm{s}$, resulting in a covered distance of $533 \mathrm{~m}$.

\subsection{Gap}

With its flippers extended, the OT- 4 traversed a gap $49 \mathrm{~cm}$ (19.25 in.) wide, as shown in Figure 27. This is $52 \%$ of its nominal length. On closer inspection of Figure 27, and based on geometry and center of mass (c.g.) considerations alone, one might conclude that the robot could span an even wider gap. However, in several attempts the raised front segments lifted the c.g. up so much that the robot rolled over and the attempt failed. Figure 27 shows the widest gap the robot traversed without rolling over. 


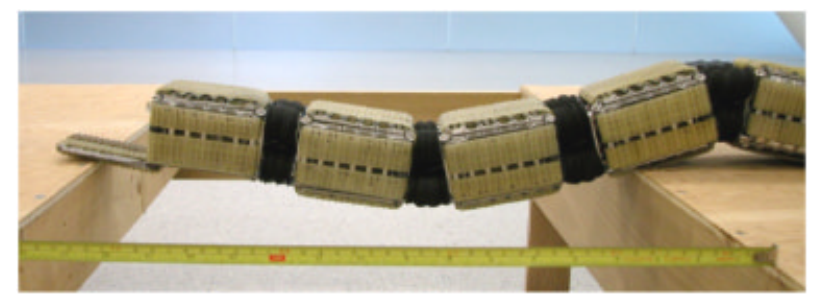

Figure 27. OT-4 traversing a gap $49 \mathrm{~cm}$ (19.25 in.) wide. This is $52 \%$ of its nominal length.

\subsection{Rockbed}

Figure 28 shows the OT- 4 during a traverse of the Rockbed Challenge. The OmniTread's "tracks-allaround" design shines in that test, since in the course of a traverse of the $\sim 5-\mathrm{m}$ Rockbed, the OT-4 rolled over frequently and oftentimes two or even three sides of the robot were in contact with rocks simultaneously. The OT- 4 successfully traversed the rockbed on every attempt, with the fastest time being 3:56 (min:s).

\subsection{Hole-in-wall and Knife-edge Hole}

There were two related Challenges involving the traversal of a hole in a vertical wall. In the Hole-in-wall Challenge, the robot had to enter into a short, horizontal pipe as shown in Figure 29. Once tracks were in the pipe, the pipe would support some of the robot's weight. The highest hole the OT-4 could enter

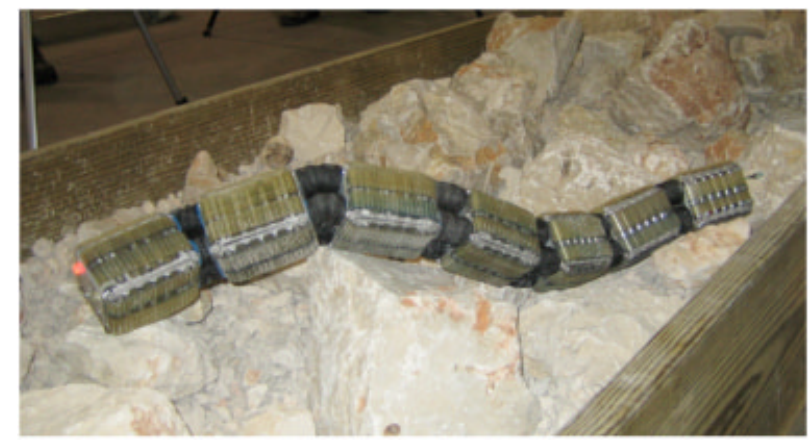

Figure 28. The OmniTread's "tracks on all sides" design shines in the Rockbed test. In a rockbed traverse, the robot inevitably rolls over frequently, and many times two or even three sides are in contact with rocks simultaneously.

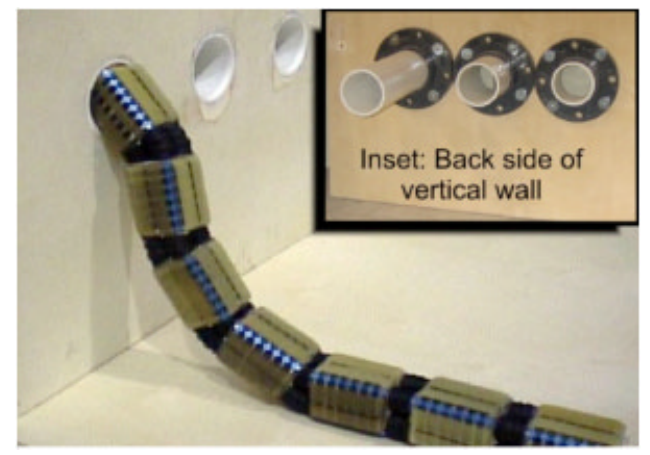

Figure 29. The Hole-in-wall Challenge at SwRI consists of a vertical wall and a horizontal, short, 4-in. diameter PVC pipe.

and traverse had its center $42 \mathrm{~cm}$ above ground. This test was performed at an earlier time, when the flipper tracks discussed in Section 4.3 were not yet implemented.

The Knife-edge Hole Challenge, depicted in Figure 30, was significantly harder to traverse. This Challenge consists of a vertical, $6 \mathrm{~mm}$ thin plywood wall with a $4 \mathrm{in}$. hole in it (the thick wooded frame holding the plywood wall obscures the side-view of the thin wall). When a joint is in the hole and the

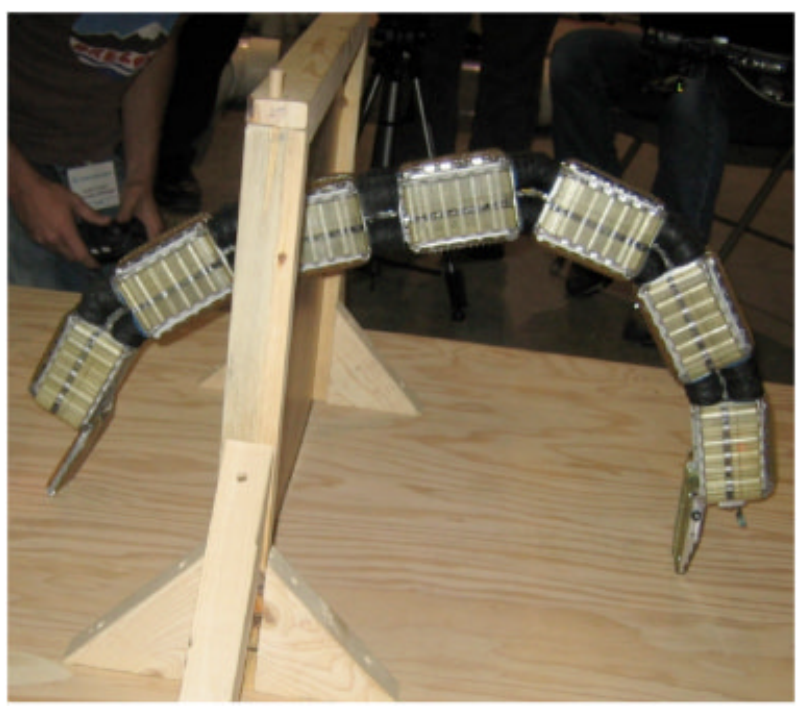

Figure 30. The Knife-edge Hole Challenge consists of a $6 \mathrm{~mm}$ thin plywood wall with a 4 in. diameter hole in it. 


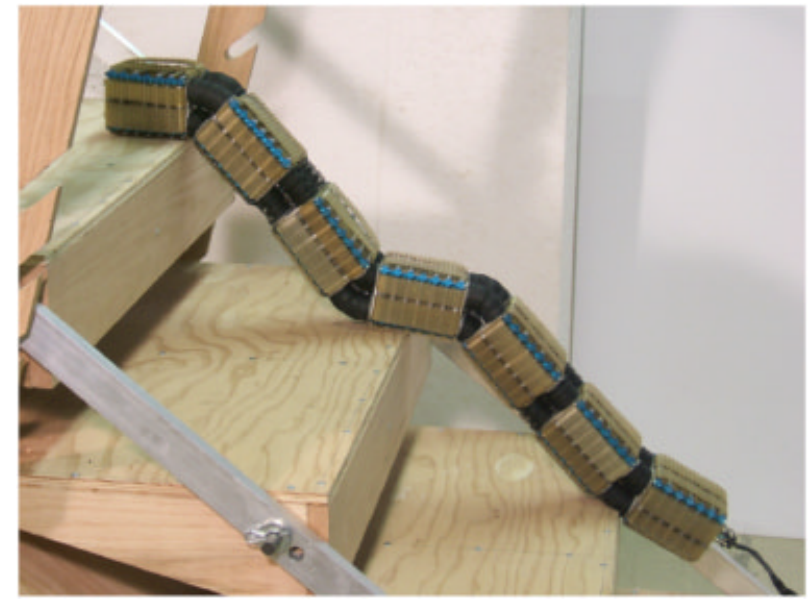

Figure 31. OT-4 climbing up a steep variable-pitch staircase set to an inclination of $40^{\circ}$.

weight of the robot is borne in part by that joint resting on the edge of the hole, then the robot is effectively stuck (recall the discussion on inert surfaces in Section 2). To avoid getting stuck, the robot has to support all of its weight on its distal segments, so as to straddle the hole and lift its inert parts entirely off the edge of the hole. The utility of the flipper tracks is obvious in Figure 30, as they extend the reach of the distal segments to the floor. The highest knife-edge hole the OT- 4 could successfully pass through this way was one that had its center $30 \mathrm{~cm}$ above ground.

\subsection{Stair Climbing}

The OT-4 performed very well on stairs. It managed consistently to climb up stairs with different combinations of rise/tread, up to an angle of $40^{\circ}$ with a rise of $21 \mathrm{~cm}\left(8 \frac{1}{4} \mathrm{in}.\right)$ and a tread of $25.4 \mathrm{~cm}$ (10 in.) as shown in Figure 31.

\subsection{Vertical Pipe Climb}

One particularly strenuous Challenge is the vertical pipe climb. It requires a great deal of strength in the joints as well as a very powerful drive train. In a vertical climb, the joints have to press the appropriate segments against the inside wall of the pipe with great torque so as to generate large normal forces at the points of contact between the segment and the

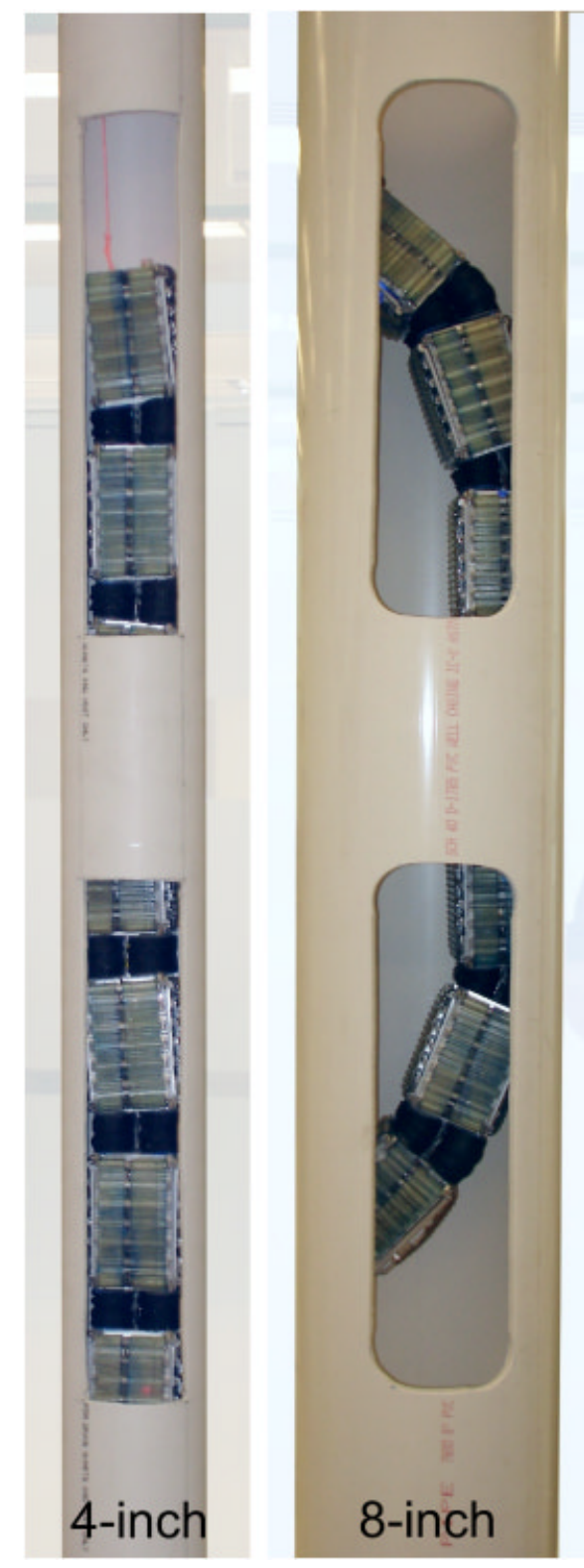

Figure 32. OT-4 climbing up vertically inside a $4 \mathrm{in}$. (left) and an 8 in. (right) diameter PVC pipe. The cord visible in the left picture is a safety line (belay), to protect it case of fall. The belay was not used to pull the robot. The "windows" in the opaque PVC pipe reveal the pose and the progress of the robot during these tests, but they did not interfere with the runs.

pipe wall. Similarly, the drive train has to produce enough torque to support the full weight of the robot, whereas under most other drive conditions on horizontal or sloped terrain the required drive 
torque is much smaller. Figure 32 shows the OT- 4 climbing up in a $4 \mathrm{in}$. and in an $8 \mathrm{in}$. insider diameter PVC pipe. The longest vertical pipe that we can support in our lab is $2.30 \mathrm{~m}$. The OT-4 climbed the available $1.36 \mathrm{~m}$ in these pipes at average speeds of $8 \mathrm{~cm} / \mathrm{s}$ in the 4 in. pipe and $6 \mathrm{~cm} / \mathrm{s}$ in the 8 in. pipe. In tests at SwRI, where much longer vertical pipes are used, the OT-4 climbed up successfully in vertical 4 and 6 in. diameter pipes. In the 4 in. pipe the OT- 4 climbed $5.04 \mathrm{~m}$ at $4.3 \mathrm{~m} / \mathrm{s}$ and in the 6 in. pipe the robot climbed $4.48 \mathrm{~m}$ at $4.4 \mathrm{~cm} / \mathrm{s}$.

\subsection{Other Tests}

The OT-4 was tested at SwRI and at our own lab in numerous other Challenges. The robot was successful in some of these tests and had limited success in others. Tests in which the OT-4 performed poorly were driving in deep sand and through underbrush. In both environments the OT-4 almost finished the prescribed course, but suffered part breakages that could not be overcome without ending the experiment. The cause of these failures is that sand, twigs, or other small debris is ingested into the tracks and all of the drive system. It is interesting to note, though, that the larger and heavier OT- 8 performed exceptionally well on those exact same terrains, completing each of multiple runs successfully and in a short time. We conclude from this performance comparison that in tracked drive systems, there is a minimal ratio between the drive sprocket diameter and the diameter of environmental particles that needs to be met in order to traverse the terrain successfully. It may be possible to harden components further, and thereby avoid the breakages, but it was clear in both environments that large stresses acted on the drive system due to the ingested debris.

Because of the OT-4's poor performance in sand (due to sand being ingested into the drive system but not due to track sinkage), we omit in this paper any further analysis related to track-sand interaction.

\section{CONCLUSIONS}

This paper describes the design and features of the "OT-4" serpentine robot, which is part of the family of so-called "OmniTread" robots that were built at our lab between 2001 and 2006. In contrast to the earlier OmniTread OT-8, the smaller OT-4 is designed to carry on-board energy resources (electric batteries and two miniature air compressors) for up to $75 \mathrm{~min}$ of untethered operation.

Unique and innovative features of the OT-4 are (1) joint actuation with pneumatic bellows, (2) body surrounded by extra-wide tracks on all sides, (3) a single drive motor powering all tracks, (4) microclutches that can engage/disengage each track individually, and (5) extendable flipper tracks in the head and segment.

The OT-4 performs particularly well on rugged but hard terrain, such as the rubble of a collapsed building (for which it was originally designed), rocks, or gravel. The OT-4 also performs very well climbing over obstacles, climbing up stairs, climbing into or through holes, or driving through pipes. Highly unfavorable combinations of Challenges that the OT- 4 could overcome individually, however, could prove unsurpassable. The OT-4 performed less well on terrain covered deeply by debris with characteristic diameter smaller than, say, $3 \mathrm{~mm}$. Examples for such debris are sand, dirt, and twigs. This is, however, not a flaw of the OmniTread design in general, because our larger OmniTread OT-8 performed exceptionally well on exactly those terrains that the OT- 4 had difficulties with. We also believe that we can design a dust-proof sealed version of the OT-4, which would likely fare much better in sandy terrain.

\section{ACKNOWLEDGMENTS}

This work was funded by the U.S. Department of Energy under Award No. DE FG52 2004 NA25587 as well as by the Intelligence Technology Innovation Center under Grants \#F013596 and F013019.

The authors are grateful to the OmniTread OT-4 development team for their full-time efforts: Russ Miller, Hung Nguyen, Jack Phan, Jim Berry, Justin Tesmer, Oke Liimatta, and John Baker. We are also grateful to David Thomas, Jenny Weinberg, and the many machinists who worked on the OT-4 parttime.

We are further grateful to Dr. William Hutchison and Dr. Betsy Constantine who developed the "7G" computerized learning program. This program produced the sophisticated and complex motion strategy used for traversing the steep staircase in Figure 31.

We also express our gratitude to Bill McBride 
and his team at the Southwest Research Institute (SwRI) for conducting the objective third-party tests with our OmniTread robots.

\section{REFERENCES}

Aoki, T., Ohno, H., \& Hirose, S. (2002). Design of slim slime robot II (SSR-II) with bridle bellows. Paper presented at the International Conference on Intelligent Robots and Systems (IEEE/RSJ'02), EPFL, Switzerland.

Blitch, J. G. (2003). Adaptive mobility for rescue robots. Paper presented at the SPIE Sensors, and Command, Control, Communications, and Intelligence (C3I) Technologies for Homeland Defense and Law Enforcement II Conference, Orlando, FL.

Choset, H. (2005). Video Proceedings of the 2005 IEEE International Conference on Robotics and Automation, Barcelona, Spain.

Dowling, K. (1997). Limbless locomotion: learning to crawl with a snake robot. Doctoral dissertation, Robotics Institute, Carnegie Mellon University, Pittsburgh, PA.

Granosik, G., \& Borenstein, J. (2005). Integrated joint actuator for serpentine robots. IEEE Transactions on Mechatronics, 10(5), 139-148.

Granosik, G., \& Borenstein, J. (2004). Minimizing air consumption of pneumatic actuators in mobile robots. Paper presented at the IEEE International Conference on Robotics and Automation (ICRA'04), New Orleans, LA.

Granosik, G., Hansen, M., \& Borenstein, J. (2005). The OmniTread serpentine robot for industrial inspection and surveillance. International Journal on Industrial Robots, Special Issue on Mobile Robots, 32(2), 139-148.

Hirose, S., \& Morishima, A. (1990). Design and control of a mobile robot with an articulated body. The International Journal of Robotics Research, 9(2), 99-113.

Hirose, S. et al. (1991). Design of practical snake vehicle: articulated body mobile robot KR-II. Fifth Interna- tional Conference on Advanced Robotics, Robots in Unstructured Environments, Pisa, Italy.

Kamegawa, T. et al. (2004). Development of the snake-like rescue robot KOHGA. Paper presented at the IEEE International Conference on Robotics and Automation (ICRA '04), New Orleans, LA.

Kimura, H., \& Hirose, S. (2002). Development of Genbu: active wheel passive joint articulated mobile robot. Paper presented at the International Conference on Intelligent Robots and Systems (IEEE/RSJ'02), Sept. 30-Oct. 4, EPFL, Switzerland.

Klaassen, B., \& Paap, K. L. (1999). GMD-SNAKE2: a snakelike robot driven by wheels and a method for motion control. Paper presented at the IEEE International Conference on Robotics and Automation, Detroit, MI.

Mori, M., \& Hirose, S. (2002). Three-dimensional serpentine motion and lateral rolling by active cord mechanism ACM-R3. Paper presented at the International Conference on Intelligent Robots and Systems (IEEE/ RSJ'02), EPFL, Switzerland.

Muth, J., \& Grant, E. (2000). A safer way to search disaster sites. IEEE Robotics \& Automation Magazine, September, 56-57.

Ohno, H., \& Hirose, S. (2000). Study on slime robot (proposal of slime robot and design of slim slime robot). Paper presented at the IEEE/RSJ International Conference on Intelligent Robots and Systems (IROS 2000), Takamatsu, Japan.

Osuka, K., \& Kitajima, H. (2003). Development of mobile inspection robot for rescue activities: MOIRA. Paper presented at the International Conference on Intelligent Robots and Systems (IEEE/RSJ'03), Las Vegas, NV.

Paap, K. L. et al. (2000). A robot snake to inspect broken buildings. Paper presented at the 2000 IEEE/RSJ International Conference on Intelligent Robots and Systems (IROS'00), Takamatsu, Japan.

Takayama, T., \& Hirose, S. (2000). Development of Souryu-I connected crawler vehicle for inspection of narrow and winding space. Paper presented at the 26th Annual Conference of the IEEE Industrial Electronics Society, IECON 2000, Nagoya, Aichi, Japan. 